\title{
COARSENING IN HIGH ORDER, DISCRETE, ILL-POSED DIFFUSION EQUATIONS *
}

\author{
CATHERINE KUBLIK ${ }^{\dagger}$
}

\begin{abstract}
We study the discrete version of a family of ill-posed, nonlinear diffusion equations of order $2 n$. The fourth order $(n=2)$ version of these equations constitutes our main motivation, as it appears prominently in image processing and computer vision literature. It was proposed by You and Kaveh as a model for denoising images while maintaining sharp object boundaries (edges). The second order equation $(n=1)$ corresponds to another famous model from image processing, namely Perona and Malik's anisotropic diffusion, and was studied in earlier papers. The equations studied in this paper are high order analogues of the Perona-Malik equation, and like the second order model, their continuum versions violate parabolicity and hence lack well-posedness theory. We follow a recent technique from Kohn and Otto, and prove a weak upper bound on the coarsening rate of the discrete in space version of these high order equations in any space dimension, for a large class of diffusivities. Numerical experiments indicate that the bounds are close to being optimal, and are typically observed.
\end{abstract}

Key words. Backward diffusion equations, coarsening, image processing, Perona-Malik model, You-Kaveh model.

AMS subject classifications. 35G20, 35K55.

\section{Introduction}

The well-known model of Perona and Malik [20, 21] constitutes the first instance of a nonlinear partial differential equation (PDE) arising in image processing and computer vision applications. Perona and Malik's goal was to devise a method for gradually simplifying (or coarsening) an image by diffusing out its details, starting with the smallest scales. An important point was to keep object boundaries (i.e. edges) in the image - where the image intensity is expected to be discontinuous or rapidly changing - sharp during the diffusion, until their abrupt disappearance at some point in the coarsening process. For this purpose, they introduced the following nonlinear second order parabolic PDE:

$$
u_{t}=\nabla \cdot(g(|\nabla u|) \nabla u) \text { subject to } u(x, 0)=f(x),
$$

where the initial condition $f$ is the given image to be simplified. In this model $g$ is a bounded, non-increasing positive function such that $\lim _{x \rightarrow \infty} g(x)=0$ and $g(0)=1$. It is easy to understand how the diffusion becomes spatially adaptive: if the image gradient is small, $g$ will be close to one and the image will diffuse at this location. On the other hand, if the image gradient is large (e.g., close to an edge), $g$ will be close to zero, and hence will almost stop the diffusion and keep the edges sharp. In their papers [20, 21], Perona and Malik proposed and experimented with two choices of $g$ :

$$
\begin{aligned}
g(x) & =e^{-\left(\frac{x}{b}\right)^{2}}, \\
\text { and } g(x) & =\frac{1}{1+\left(\frac{x}{b}\right)^{2}},
\end{aligned}
$$

\footnotetext{
${ }^{*}$ Received: July 24, 2009; accepted (in revised version): November 20, 2009. Communicated by Markos Katsoulakis.

${ }^{\dagger}$ Department of Mathematics, University of Michigan, Ann Arbor, USA (mareva@umich.edu). http://www-personal.umich.edu/ mareva
} 
where $b$ is a constant that acts like a contrast threshold to be chosen by the user. The second choice of $g$ given in (1.2b) is the most commonly used diffusivity in applications of the model, and constitutes what is typically meant by the Perona-Malik model in the literature.

The time evolution of the (discretized) PDE generates "cartoon-like" images that get simpler with time, and yet keeps edges in the image sharp as was intended. Unfortunately, the Perona-Malik model turns out to be ill-posed. To see this, consider (1.1) in one space dimension

$$
u_{t}=\left(R\left(u_{x}\right)_{x}\right),
$$

where $R(x)=x g(|x|),(R: \mathbb{R} \longmapsto \mathbb{R})$, and $g$ is as in (1.2). Expanding the right hand side we obtain

$$
u_{t}=R^{\prime}\left(u_{x}\right) u_{x x}
$$

which becomes backwards parabolic when $R^{\prime}\left(u_{x}\right)<0$. For the two choices of $g$ given in (1.2), $R$ is non increasing whenever the gradient is large. Thus, in the regions of the image with large gradient, the process can be interpreted as a backward heat equation. The situation is the same in higher space dimensions. The ill-posedness signifies that (1.1) may not have a solution in general. A very practical implication of this ill-posedness is that similar initial images may lead to completely divergent evolutions and hence very dissimilar output images at the end of the processing.

Consequently, there is no complete well-posedness theory for (1.1) despite many efforts towards establishing rigorous results $[4,9,10,11,15]$. Another active line of research towards understanding (1.1) considers its discrete in-space version $[6,7,8]$, while others focus on studying its regularizations in order to obtain a well-posed equation $[1,2,3,5,18]$. Nevertheless, in practice, the Perona-Malik equation is much better behaved than expected. One significant practical caveat of the model however is the staircasing artifact which produces "blocky" images: regions of moderately large gradient in the image develop flat regions separated by spurious edges. In fact, the staircasing instability of the Perona-Malik model is best explained in the one dimensional case where the solution $u$ develops steps which merge together to create larger steps as the solution evolves in time. The result is an image that looks "blocky".

You and Kaveh [24] introduced a nonlinear, fourth-order PDE as an improvement of the Perona-Malik model, particularly with regard to the staircasing artifact. In their model, the image gradually turns into a simpler and simpler piecewise linear image (as opposed to piecewise constant), which greatly abates the staircasing effect. Their fourth order PDE is

$$
u_{t}=-\Delta(R(\Delta u)) \text { subject to } u(x, 0)=f(x),
$$

where $R(x)=x g(|x|)$ and $g$ is the same nonlinear diffusivity as utilized in the PeronaMalik model. Like the Perona-Malik evolution, (1.3) generates coarser resolution images from a given one taken as initial condition, while preserving sharp contours and smoothing everything else. Numerical simulations with this PDE give similar results to Perona and Malik's in terms of noise removal and edge preservation but the staircasing artifact is almost completely absent.

However, being the fourth order analogue of the Perona-Malik model, the YouKaveh model inherits the same ill-posedness issues as its progenitor. Some analysis of this PDE has been carried out by Greer and Bertozzi in [13], where they show that 
smooth traveling wave solutions of the one dimensional You-Kaveh PDE do not exist for sufficiently large jump heights. Their conclusion conjectures that the You-Kaveh equation has finite time singularities in $u_{x x}$, just as the Perona-Malik equation has finite time singularities in the slope $u_{x}[12,14]$.

Motivated by You and Kaveh's fourth order analogue of the Perona-Malik model, we consider the following family of $2 n$-th order nonlinear diffusion equations $\left(n \in \mathbb{N}^{*}\right)$ :

$$
u_{t}=\left\{\begin{aligned}
-\Delta^{\frac{n}{2}}\left(R\left(\Delta^{\frac{n}{2}} u(x)\right)\right) & \text { if } n \text { is even, } \\
\nabla \cdot \Delta^{n-1} R\left(\nabla \Delta^{\frac{n-1}{2}} u(x)\right) & \text { if } n \text { is odd. }
\end{aligned}\right.
$$

For $n=1$ equation (1.4) becomes the well-known Perona-Malik model, and for $n=2$ we recover the You-Kaveh model. We prove rigorous upper bounds on the coarsening rate of (1.4) in any dimension $d \in \mathbb{N}^{*}$, for a large class of diffusivities $R$ that include the original choices of Perona-Malik and You-Kaveh.

Upper bounds on the coarsening rate of second order, discrete, ill-posed diffusion equations (based on the Perona-Malik model) were obtained in [7] and improved in [8] for a large class of diffusivities. In this paper we consider the same class of diffusivities as in [8] and prove analogous bounds for the $2 n$-th order equation (1.4) in any space dimension. However, our results and arguments differ from $[7,8]$ in several ways. First of all, in the present paper we work in the more general $2 n$-th order setting. Secondly, the rigorous bounds in $[7,8]$ are stated in terms of the decrease rate of the energy of the system (a somewhat abstract quantity), whereas the real quantity of interest in applications is the edge density in the solutions (equivalently, the number of "spikes" in its derivative). Indeed, the numerical experiments - unlike the rigorous results - presented in these papers are in terms of the spike density in the derivative of the solution, and even though the spike density and the energy can be related to each other through a heuristic argument, it is desirable to have a completely rigorous statement directly in terms of the spike density of the solution. In the present paper, we replace the heuristic step by a rigorous argument and therefore obtain rigorous bounds for the spike density. Third, unlike in the second order case, the high order models we consider lack a maximum principle, which requires certain arguments to be modified. In particular, the construction of the test function needed to estimate the bounds in the low dimensional case is different from the construction used in $[7,8]$. Our construction is simple and more general than the one proposed in these previous papers. Finally, it should be mentioned that the discrete PDEs considered in $[7,8]$ are only related to the Perona-Malik scheme in one space dimension; in higher dimensions, the correspondence between the image processing model and the PDEs considered in the afore-mentioned papers is lost. In this paper, the fourth order PDEs considered and the theory developed for them corresponds to the relevant image processing model - the You-Kaveh model - in all space dimensions. For the general $2 n$-th order PDEs, the correspondence between the theory and the model exists in all space dimensions whenever $n$ is even.

As in the second order case, the high order discrete-in-space equations exhibit an instability that very rapidly leads to the formation of spikes or discontinuities of width exactly one grid point, starting from generic initial data. These singular structures then interact without changing their location: small ones get absorbed by larger ones, leading to fewer and larger structures and a corresponding increase in the length scale of the solution as measured by e.g. the average distance between the structures. We call the rate of increase of this quantity the coarsening rate.

In computer vision and image processing applications, the coarsening of the You- 
Kaveh flow can be observed as the noisy image gradually simplifies into a piecewise linear approximation by merging neighboring regions and preserving their edges. The rate at which this coarsening takes place is therefore crucial since it could provide a way to automatically stop the evolution at a given level of simplification in the image. Moreover, such an analysis would provide insight into the effect of different diffusivities on the dynamics. Our method is based on a recent technique developed by Kohn and Otto [16] for energy driven systems. This method uses the energy $E$ of the system and a length scale quantity $L$ in two inequalities: a dissipation inequality that involves $\frac{d L}{d t}$ and $\frac{d E}{d t}$, and an interpolation inequality between $L$ and $E$. Combined with an ODE argument, these inequalities lead to a lower bound on the time-average of the energy which is equivalent to an upper bound on the coarsening rate. We present our results in any space dimension. For simplicity and clarity purposes, we present a detailed study of the fourth order case (corresponding to the You-Kaveh model from image processing), and then indicate how the results extend to higher order analogues.

The paper is organized as follows. In section 2 we focus on the analysis of the YouKaveh equation, and describe some of the fundamental properties of the corresponding scheme. In particular, we show that in our setting the only stable stationary solution is the one with a single spike; the dynamics therefore typically does not halt until a very coarse configuration is reached. In section 3, we establish our main result on coarsening for the You-Kaveh model and present its proof in any space dimension. Section 4 presents the general result on coarsening for the $2 n$-th order equation, and outlines its proof. In section 5 we show numerical experiments that corroborate our estimates, and conclude in section 6 .

\section{Analysis of the You-Kaveh model}

2.1. The equations. We consider the fourth order ill-posed nonlinear PDE proposed by You and Kaveh [24]

$$
u_{t}=-\Delta(g(\Delta u) \Delta u)
$$

and let $R(s)=s g(s)$, so that equation (2.1) becomes

$$
u_{t}=-\Delta(R(\Delta u))
$$

If we let $v=\Delta u$ and take the Laplacian of equation (2.2), we obtain the following equation for $v$ :

$$
v_{t}=-\Delta^{2}(R(v))
$$

In the rest of this section, we will study the discrete version of equation $(2.3)$ on $[0,1]^{d}$ for a large class of diffusivities $R$, where $d \in \mathbb{N}^{*}$ is the spatial dimension.

2.2. The scheme. We work on a uniform discretization of the domain $[0,1]^{d}$ and let $\delta=\frac{1}{N}$ denote the grid size in each of the coordinate directions. Consider the unit-spaced lattice $\mathbb{L}=\{0,1, \cdots, N-1\}^{d}$ and let $i \in \mathbb{L}$ be the vector $\left(i_{1}, \cdots, i_{j}, \cdots, i_{d}\right) \in \mathbb{L}$ corresponding to the point $\left(\frac{i_{1}}{N}, \cdots, \frac{i_{j}}{N}, \cdots, \frac{i_{d}}{N}\right)$ in $[0,1]^{d}$. Define the discrete Laplacian operator $\Delta_{\delta}$ operating on the grid function $v$ at the point $i \in \mathbb{L}$ to be

$$
\Delta_{\delta} v_{i}:=\sum_{j=1}^{d} D_{j, \delta}^{+} D_{j, \delta}^{-} v_{i},
$$


where

$$
D_{j, \delta}^{+} v_{i}:=\frac{v_{i+e_{j}}-v_{i}}{\delta}=\frac{v_{i_{1}, \cdots, i_{j}+1, \cdots, i_{d}}-v_{i_{1}, \cdots, i_{j}, \cdots, i_{d}}}{\delta},
$$

and

$$
D_{j, \delta}^{-} v_{i}:=\frac{v_{i}-v_{i-e_{j}}}{\delta}=\frac{v_{i_{1}, \cdots, i_{j}, \cdots, i_{d}}-v_{i_{1}, \cdots, i_{j}-1, \cdots, i_{d}}}{\delta},
$$

are forward and backward difference quotients respectively, in the $j$ th coordinate direction, for $1 \leq j \leq d$. The "fourth order" discrete system that we consider is the following natural spatial finite difference discretization of $(2.3)$

$$
\frac{d v_{i}}{d t}=-\Delta_{\delta}^{2}\left(R\left(v_{i}\right)\right), i \in \mathbb{L} .
$$

This discrete in space, continuum in time version of (2.3) is equivalent to the discretization of the You-Kaveh model used in practice in image processing applications.

We impose periodic boundary conditions on (2.4), which means that we identify the lattice $\mathbb{L}$ with $\mathbb{Z}^{d}$ on which for any integer $0 \leq i_{j} \leq N-1$ and $k_{j} \in \mathbb{Z}, 0 \leq j \leq N-1$, the point $\left(i_{1}+k_{1} N, \cdots, i_{d}+k_{d} N\right)$ is identified with $\left(i_{1}, \cdots, i_{d}\right)$. This periodicity ensures that the dynamics of (2.4) leave the total mass

$$
\mu:=\frac{1}{N^{d}} \sum_{i \in \mathbb{L}} v_{i}
$$

unchanged. This system also has a non-increasing energy

$$
E(v)=\frac{1}{N^{d}} \sum_{i \in \mathbb{L}} f\left(v_{i}\right),
$$

where the density function $f$ is defined as

$$
f(x)=\int_{0}^{x} R(\xi) d \xi
$$

for $x \in \mathbb{R}$. For smooth and strictly convex energy densities $f$, solutions generated by the scheme (2.4) would be expected to converge (as $h \rightarrow 0^{+}$) to the solution of the parabolic PDE $(2.3)$ on $[0,1]^{d}$, with periodic boundary conditions. In this paper, however, we consider non-convex even energy densities $f$ that satisfy $f(x)>\eta|x|^{\alpha}$ for some constant $\eta>0, \alpha \in[0,1[$, and all $|x|$ large enough. Moreover, for $x \geq 0$, the functions $f$ will be assumed to be strictly convex near 0 , concave for large $x$, and have only one inflection point $b>0$. By symmetry, $\pm b$ are the two inflection points of $f$ on $\mathbb{R}$. Since (2.3) can be expanded as

$$
v_{t}=-\Delta^{2}(R(v))=-R^{\prime}(v) \Delta^{2} v+\underbrace{F\left(v, \nabla v, \nabla^{2} v, \nabla^{3} v\right)}_{\text {Lower Order Terms }},
$$

we see that equation (2.3) becomes backward parabolic whenever $R^{\prime}(v)<0$, which occurs whenever $|v|$ is large enough. Consequently, there is no complete well-posedness theory for equation (2.3). Furthermore, we make the following assumptions on $R$ :

1. $R: \mathbb{R} \rightarrow \mathbb{R}$ is smooth, 
2. $R^{\prime}(x)>0$ on $[-b, b[$,

3. $R^{\prime}(x)<0$ on $]-\infty,-b[\bigcup] b, \infty[$, and

4. $R(0) \leq \lim _{x \rightarrow \pm \infty} R(x)$.

In image processing applications, a common choice for the diffusivity $R$ is

$$
R(x)=\frac{x}{1+\left(\frac{x}{b}\right)^{2}},
$$

which corresponds to the choice of $g$ given in (1.2b). The corresponding density function $f$ of $(2.8)$ is the logarithmic density function

$$
f(x)=\frac{b}{2} \ln \left(1+\left(\frac{x}{b}\right)^{2}\right), b>0 .
$$

However, other choices of $R$ leading to backwards parabolic behavior are regularly used in applications. The practical implications of the choice of the diffusivity $R$ on results has often been raised in the engineering literature (see e.g. [22]) and is still a current topic of research. As an illustration, figure 2.1 shows the graph of $f, R$ and $R^{\prime}$, where the density function $f$ is as in (2.9).

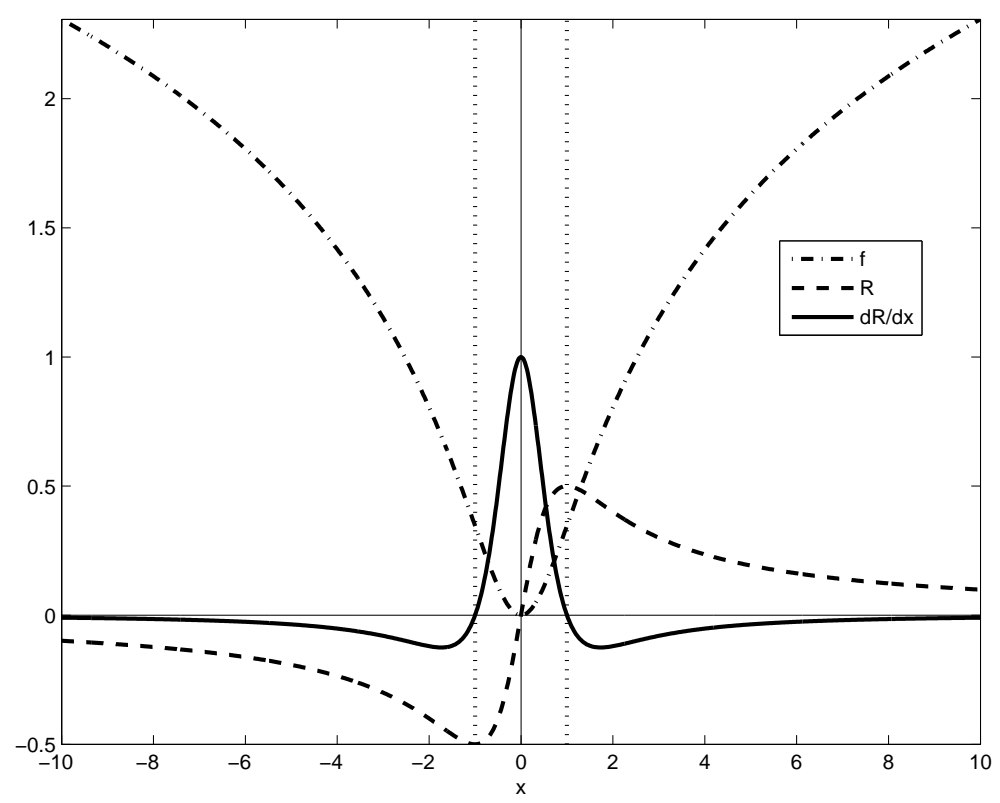

FIG. 2.1. Functions $f, R$ and $R^{\prime}$ with $R(x)=\frac{x}{1+x^{2}}$ and $f(x)=\frac{1}{2} \ln \left(1+x^{2}\right)$. The changes in concavity of the function $f$, which occur at \pm 1 , are shown by the two dotted vertical lines.

2.3. Terminology and basic setup. The system (2.4) is gradient descent for the energy $E$ defined in (2.6) with respect to the discrete $H^{-2}$ norm. This norm can be expressed by duality as

$$
\|v\|_{H^{-2}}:=\left\{\sup _{\phi} \frac{1}{N^{d}} \sum_{i \in \mathbb{L}}\left(v_{i}-\mu\right) \phi_{i}: \frac{1}{N^{d}} \sum_{i \in \mathbb{L}}\left(\Delta_{\delta}\left(\phi_{i}\right)\right)^{2} \leq 1\right\} .
$$


If the initial data of (2.4) satisfies $\left.v_{i} \in\right]-b, b$ [ so that it lies completely in the forward parabolic regime, the evolution proceeds as a typical parabolic smoothing, at least for small time. If on the other hand the initial data's mean value $\mu$ satisfies

$$
|\mu|>b \text {, }
$$

then part of the mass of the data sits in the ill-posed regime $]-\infty,-b[\cup] b, \infty[$ for all time, due to the conservation of mass. In this case, instabilities in the form of spikes rapidly develop from the initial data and start to interact, gradually decreasing in number but growing in size. These spikes are located at the grid points where the solution exceeds the parabolicity threshold $b$. We therefore use the following terminology:

There is a spike at the $j$-th grid point if $\left|v_{j}\right| \geq b$.

Each spike is supported by one grid-point and remains at that grid location during the entire evolution of the equation. However, while the location of each spike is fixed, their height varies. Each spike either grows, or shrinks, and may eventually disappear as its mass is absorbed by others. Indeed, the concavity of $f$ on $]-\infty,-b[\bigcup] b, \infty[$ encourages the accumulation of mass into fewer and higher spikes during the evolution. Smaller spikes thus get absorbed into larger ones until, generically, only one spike containing most of the mass remains; this is what we mean by coarsening of the system in this paper. An example of this coarsening is shown in figure 2.2. During the evolution, the average distance between spikes is observed to increase, and constitutes a natural length scale for the system. The length scale $L$ should be inversely proportional to the number of spikes $K$ and therefore should behave something like

$$
L \sim \frac{1}{K^{\beta}},
$$

where $\beta>0$ may depend on the spatial dimension. In addition, since $f$ is an increasing function of $|v|$ for $v \in \mathbb{R}$, most of the energy is initially contained in the spikes, which suggests

$$
E \sim \frac{K^{\gamma}}{N^{d}}
$$

where $\gamma>0$. Both $\gamma$ and $\beta$ are parameters to be identified. The interpolation inequality that we need relates the energy $E$ and the length scale $L$ in a one-sided version of

$$
E L^{\frac{\gamma}{\beta}} \sim C(N),
$$

where $C(N)$ is a constant depending only on $N$. So if we had a length scale $L$ that behaved as in equation (2.11), we could then use the relation (2.12) to immediately have the interpolation inequality

$$
E L^{\frac{\gamma}{\beta}} \sim \frac{1}{N^{d}} .
$$

A natural choice for the length scale quantity $L$ is therefore $L=\frac{1}{K^{\beta}}$, but the decay relation would then be hard to prove since it relates $\frac{d L}{d t}$ and $\frac{d E}{d t}$, and thus would involve differentiating $L=\frac{1}{K^{\beta}}$ which is a discrete function of time. For these reasons, we choose

$$
L=\|v\|_{H^{-2}},
$$




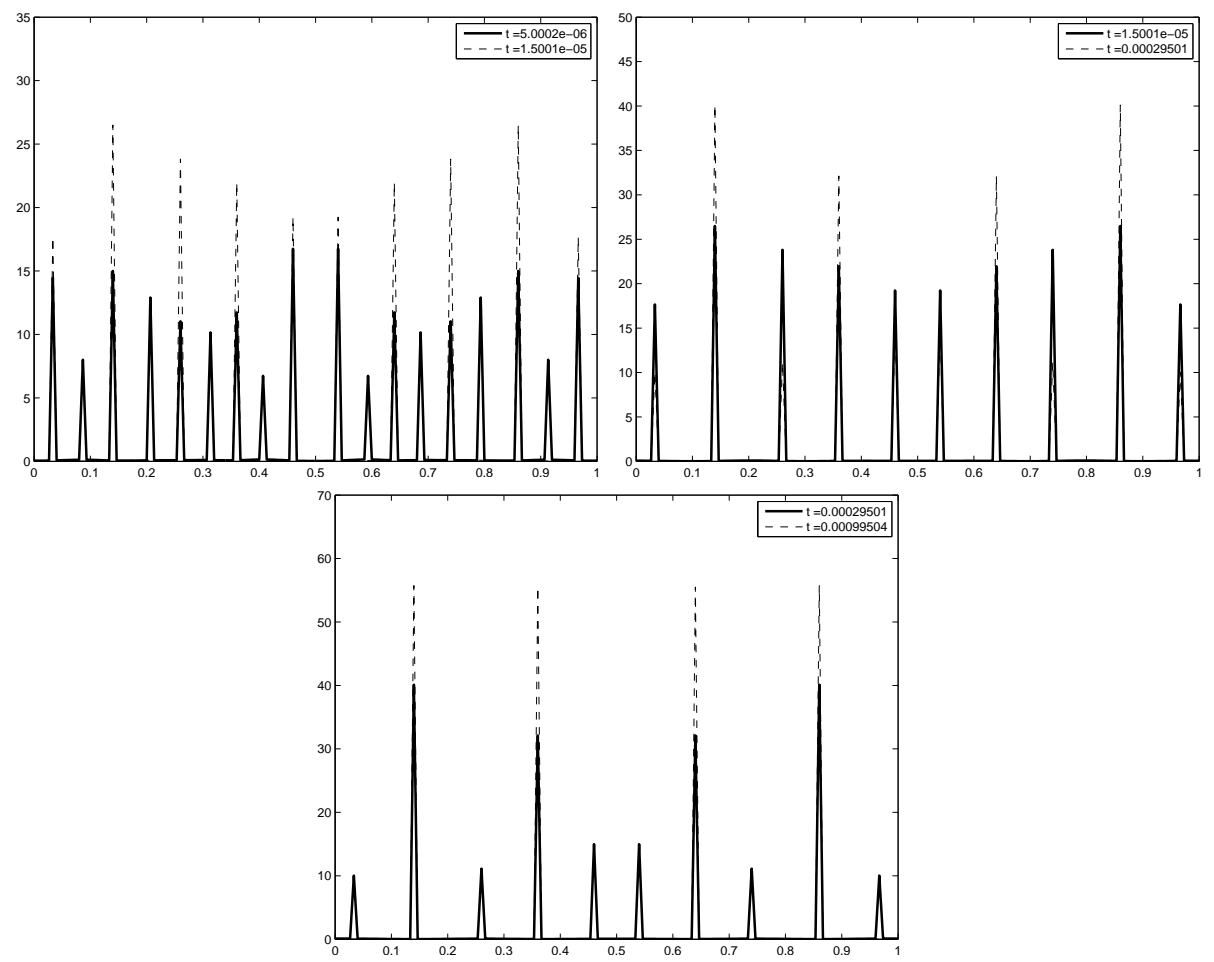

FIG. 2.2. One dimensional evolution of (2.4) for $N=150$ and initial solution $v_{i}(0)=\frac{3}{2}+$ $10^{-3} \sin (\pi x)$ with $x \in[0,1]$. The solution quickly forms spikes whose number decreases as some of them shrink and disappear, while others grow taller to preserve the total mass.

which is motivated by the fact that the system (2.4) is gradient descent for the energy $E$ defined in (2.6) with respect to the discrete $H^{-2}$ norm. This choice is also similar to length scales used in $[7,16,17,19]$. In particular, $L$ as given in (2.13) will imply the following scaling between the length scale $L$ and the number of spikes $K$

$$
L \sim \begin{cases}\frac{1}{K_{1}^{\frac{2}{2}}} & \text { for } 1 \leq d \leq 3 \\ \frac{\sqrt{K}}{\sqrt{K}} & \text { for } d \geq 4\end{cases}
$$

In the next section, we will study the stability of all the steady state solutions of the one dimensional system in the case of the logarithmic density function (2.9). The results show that solutions with more than two spikes, together with the constant solution equal to $b$ or $-b$ (depending on the sign of $\mu$ ), are unstable. The single spike solution (obtained when $|\mu|>b$ ) and the constant solution with values in $]-b, b[$ (obtained when $|\mu|<b$ ) are both stable. We note that these stability results can easily be generalized to the more general density functions $f$ that are considered in this paper.

2.4. Stability of stationary states. In the one dimensional case, the scheme becomes

$$
\frac{d v_{i}}{d t}=-\left(D_{\delta}^{+} D_{\delta}^{-}\right)^{2}\left(R\left(v_{i}\right)\right), \quad 0 \leq i \leq N-1,
$$


where

$$
D_{h}^{+} v_{i}=\frac{v_{i+1}-v_{i}}{\delta}
$$

and

$$
D_{h}^{-} v_{i}=\frac{v_{i}-v_{i-1}}{\delta}
$$

are forward and backward difference quotients.

2.4.1. Steady states. In order to find the steady states of $(2.14)$ we need to solve

$$
\frac{d v_{i}}{d t}=0, \quad \forall i \in[0, \cdots, N-1]
$$

i.e.,

$$
\left(D_{\delta}^{+} D_{\delta}^{-}\right)^{2}\left(R\left(v_{i}\right)\right)=0, \quad \forall i \in[0, \cdots, N-1] .
$$

Using the periodic boundary conditions we obtain

$$
R\left(v_{i}\right)=C, \quad \forall i \in[0, \cdots, N-1],
$$

where $C \in \mathbb{R}$ is a constant. Since the range of $s \mapsto R(s)=\frac{s}{1+\left(\frac{s}{b}\right)^{2}}$ on $\mathbb{R}$ is $\left[-\frac{b}{2}, \frac{b}{2}\right]$ we see that $-\frac{b}{2} \leq C \leq \frac{b}{2}$. We now consider three different cases:

1. $\mathbf{C}=\mathbf{0}$

Then $v_{i}=0 \forall i \in[0, \cdots, N-1]$, which violates the conservation of mass (2.5)

for $\mu \neq 0$. Thus if $\mu \neq 0, v=0$ is not a feasible solution.

If $\mu=0$ however, the solution $v=0$ is asymptotically stable.

2. $\mathbf{C}=\frac{\mathbf{b}}{2}$ or $\mathbf{C}=-\frac{\mathbf{b}}{2}$

(a) If $C=\frac{b}{2}$, then $v_{i}=b, \forall i \in[0, \cdots, N-1]$.

(b) If $C=-\frac{b}{2}$, then $v_{i}=-b, \forall i \in[0, \cdots, N-1]$.

3. $\mathbf{0}<\mathbf{C}<\frac{\mathbf{b}}{\mathbf{2}}$ or $-\frac{\mathbf{b}}{\mathbf{2}}<\mathbf{C}<\mathbf{0}$.

(a) If $0<C<\frac{b}{2}$, then (2.15) has two solutions that we call $v_{+}$and $v_{-}$, with

$$
0<v_{-}<b<v_{+}<\infty
$$

Also the condition $R\left(v_{-}\right)=R\left(v_{+}\right)$gives

$$
v_{+}=\frac{b^{2}}{v_{-}} .
$$

Now we define $K(1 \leq K \leq N-1)$ to be the number of grid points that have the value $v_{+}$. Conservation of mass (2.5) then implies

$$
K v_{+}+(N-K) v_{-}=N \mu .
$$

We note that for $K=0$, the steady state solution is just the constant solution with value $v_{-}=\mu$, which happens when $0<\mu<b$. Similarly, when $K=N$, the steady state is the constant solution with value $v_{+}=\mu$, 
which occurs when $\mu>b$. For $1 \leq K \leq N-1$, we use equation (2.16) and (2.17) to solve for $v_{-}$and $v_{+}$and obtain the following result:

Under the condition $\mu \geq \mu_{K, N}$ with $\mu_{K, N}=\frac{2 b \sqrt{K(N-K)}}{N}$, we have the existence of two real solutions $v_{+}$and $v_{-}$with value

$$
\left\{\begin{array}{l}
v_{+}=\frac{N \mu+\sqrt{(N \mu)^{2}-4 K(N-K) b^{2}}}{2 K} \\
v_{-}=\frac{N \mu-\sqrt{(N \mu)^{2}-4 K(N-K) b^{2}}}{2(N-K)} .
\end{array}\right.
$$

(b) If $-\frac{b}{2}<C<0,(2.15)$ has two solutions that we call $v_{+}$and $v_{-}$, with $-\infty<v_{+}<-b<v_{-}<0$. Using similar calculations we obtain the following:

For $1 \leq K \leq N-1$, and under the condition $\mu \leq-\mu_{K, N}$, we have the existence of two real solutions $v_{+}$and $v_{-}$with value

$$
\left\{\begin{array}{l}
v_{+}=\frac{N \mu-\sqrt{(N \mu)^{2}-4 K(N-K) b^{2}}}{2 K} \\
v_{-}=\frac{N \mu+\sqrt{(N \mu)^{2}-4 K(N-K) b^{2}}}{2(N-K)}
\end{array}\right.
$$

For $K=0$, the steady state solution is the constant solution with value $v_{-}=\mu$, which happens in the case $-b<\mu<0$, and for $K=N$, the steady state is the constant solution with value $v_{+}=\mu$, which occurs when $\mu<$ $-b$.

So the steady states of equation (2.14) are vectors $v$ with $K$ grid points with value $v_{+}$and $(N-K)$ grid points with value $v_{-}$, for $1 \leq K \leq N-1$. The condition on $\mu$ ensures the existence of $v_{+}$and $v_{-}$as real solutions. If $|\mu|<\mu_{K, N}$ for $1 \leq K \leq N-1$, there are no real solutions $v_{+}$and $v_{-}$. The steady state solution with $K=0$ is the constant solution $v=\mu$, which occurs when $|\mu|<b$. Similarly, the steady state solution with $K=N$ is the constant solution $v=\mu$, in the case $|\mu|>b$. Note that $\mu_{K, N} \leq b$, for all $0 \leq K \leq N . \mu_{K, N}$ is only needed for $1 \leq K \leq N-1$, but it is also defined for $K=0$ and $K=N$. In particular, $\mu_{0, N}=\mu_{N, N}=0$.

Lemma 2.1. Suppose $|\mu|>\mu_{1, N}$ and let $v_{-}$be as in (2.18) or (2.19) for $K=1$, namely

$$
v_{-}=\frac{N \mu-\sqrt{(N \mu)^{2}-4(N-1) b^{2}}}{2(N-1)} \text { or } v_{-}=\frac{N \mu+\sqrt{(N \mu)^{2}-4(N-1) b^{2}}}{2(N-1)} .
$$

Then

$$
(N-1) v_{-}^{2}<b^{2}
$$

Proof. For either $v_{-}$above, we obtain after simplification

$$
(N-1) v_{-}^{2}=\frac{2}{\frac{4(N-1)}{(N \mu)^{2}}}\left(1-\sqrt{1-\frac{4(N-1) b^{2}}{(N \mu)^{2}}}\right)-b^{2},
$$


with $0<\frac{4(N-1)}{(N \mu)^{2}}<\frac{1}{b^{2}}$. Now we consider the function $k(x)=\frac{1}{x}\left(1-\sqrt{1-b^{2} x}\right)$ where $x \in] 0, \frac{1}{b^{2}}[$. Since the range of the function $k$ is $] \frac{b^{2}}{2}, b^{2}[, \forall x \in] 0, \frac{1}{b^{2}}[$, we obtain

$$
2 k\left(\frac{4(N-1)}{(N \mu)^{2}}\right)-b^{2}=(N-1) v_{-}^{2}<b^{2},
$$

which proves the lemma.

We note that if $K=1$ and $|\mu|=\mu_{1, N}$ then $(N-1) v_{-}^{2}=b^{2}$, leading to

$$
v_{+}=b \sqrt{N-1} \text { and } v_{-}=\frac{b}{\sqrt{N-1}} \text {. }
$$

2.4.2. Stability analysis. In this section, we study the stability of the steady states using energy based arguments that easily generalize to higher dimensions. In [23], Witelski et al. studied the stability of the steady states of a similar system using linearization. Their analysis was done in the one dimensional case only.

Proposition 2.2. Equilibrium solutions with $K \geq 2$ are unstable.

For the proof, we refer to the one of Proposition 4 in Witelski et al. [23].

Proposition 2.3. For $|\mu|<b$, the equilibrium solution with $K=0$ (i.e., $v_{i}=v_{-}=\mu$, $\forall i \in[0, \cdots, N-1])$ is stable.

Proof. We prove this result by considering the energy functional (2.6)

$$
E(v)=\frac{1}{N} \sum_{i=0}^{N-1} f\left(v_{i}\right)
$$

where $f$ is as in (2.7), and checking whether any perturbation of the constant steady state $v_{-}$can result in a decrease in energy. We thus consider the minimization problem

$$
\min _{v} E(v) \text { such that } \sum_{i=0}^{N-1}\left(v_{i}-v_{-}\right)^{2} \leq \epsilon \text { and } \frac{1}{N} \sum_{i=0}^{N-1} v_{i}=v_{-}=\mu,
$$

for $\epsilon>0$. Suppose the minimum of the energy is reached at a certain $v^{m}$ which is not constant, i.e., $\exists i_{1}$ and $i_{2} \in[0, \cdots, N-1]$ such that $v_{i_{1}}^{m}$ is the smallest value, $v_{i_{2}}^{m}$ the largest value, and $v_{i_{1}}^{m} \neq v_{i_{2}}^{m}$.

Now define $\tilde{v}^{m}$ as

$$
\tilde{v}_{i}^{m}= \begin{cases}v_{i}^{m}, & \forall i \in[0, \cdots, N-1] \backslash\left\{i_{1}, i_{2}\right\} \\ v_{i_{2}}^{m}, & \text { if } i=i_{1} \\ v_{i_{1}}^{m}, & \text { if } i=i_{2} .\end{cases}
$$

Trivially $E\left(v^{m}\right)=E\left(\tilde{v}^{m}\right)$. Now we choose $\epsilon$ small enough so that none of the values of $v^{m}$ reside outside the interval $]-b, b[$, namely

$$
\left|v_{i}^{m}\right|<b, \forall i \in[0, \cdots, N-1] .
$$

Since $f$ is strictly convex on $]-b, b[$, we have

$$
f\left(\frac{v_{i}^{m}+\tilde{v}_{i}^{m}}{2}\right)=\frac{1}{2}\left(f\left(v_{i}^{m}\right)+f\left(\tilde{v}^{m}\right)\right), \forall i \in[0, \cdots, N-1] \backslash\left\{i_{1}, i_{2}\right\},
$$




$$
f\left(\frac{v_{i_{1}}^{m}+\tilde{v}_{i_{1}}^{m}}{2}\right)=f\left(\frac{v_{i_{1}}^{m}+v_{i_{2}}^{m}}{2}\right)<\frac{1}{2}\left(f\left(v_{i_{1}}^{m}\right)+f\left(v_{i_{2}}^{m}\right)\right)
$$

and similarly for $i=i_{2}$. We therefore obtain

$$
E\left(\frac{v^{m}+\tilde{v}^{m}}{2}\right)<\frac{1}{2}\left(E\left(v^{m}\right)+E\left(\tilde{v}^{m}\right)\right)=E\left(v^{m}\right)
$$

contradicting the assumption that $v^{m}$ achieves the minimum of the energy. The constant solution is therefore the only one that minimizes the energy and thus is asymptotically stable.

Proposition 2.4. Assume $N \geq 3$. For $\mu=b$, the constant equilibrium solution $v \equiv b$ is unstable. Similarly, for $\mu=-b$ the constant equilibrium solution $v \equiv-b$ is unstable.

Proof. We prove the lemma for the constant solution $v \equiv b$. The proof for $v \equiv-b$ is analogous. We choose an integer $M \in[2, N-1]$ and perturb the equilibrium solution in the following way: we increase one grid point from $b$ to $b+\epsilon$ and decrease $M$ other grid points from $b$ to $b-\epsilon / M$ where $\epsilon>0$ will be determined later. The remaining grid points have value $b$. We define $v^{0}$ to be the constant solution equal to $b$ and by $v^{\epsilon}$ the perturbed solution. We note that $\left\|v^{\epsilon}-v^{0}\right\|_{2}=\sqrt{\frac{M+1}{N M}} \epsilon$.

Now we will show that $v^{\epsilon}$ decreases the energy, i.e.,

$$
\forall M \in[2, N-1], \exists \epsilon>0 \text { such that } E\left(v^{\epsilon}\right)<E\left(v^{0}\right) .
$$

Calculating the energy for both $v^{0}$ and $v^{\epsilon}$ we obtain

and

$$
E\left(v^{0}\right)=f(b)
$$

$$
E\left(v^{\epsilon}\right)=\frac{1}{N}\left((N-M-1) f(b)+M f\left(b-\frac{\epsilon}{M}\right)+f(b+\epsilon)\right) .
$$

Subtracting $E\left(v^{0}\right)$ from $E\left(v^{\epsilon}\right)$, we obtain

$$
E\left(v^{\epsilon}\right)-E\left(v^{0}\right)=\frac{1}{N}\left(\left(M f\left(b-\frac{\epsilon}{M}\right)+f(b+\epsilon)\right)-(M+1) f(b)\right)=\frac{\zeta(\epsilon)}{N},
$$

where $\zeta(\epsilon)=\left(M f\left(b-\frac{\epsilon}{M}\right)+f(b+\epsilon)\right)-(M+1) f(b)$. obtain

We now show that $\exists \epsilon>0$ such that $\zeta(\epsilon)<0$. Replacing $f$ by its expression we

$$
\zeta(\epsilon)=\frac{b}{2} \ln \left(\left(1-\frac{\epsilon}{b M}+\frac{1}{2}\left(\frac{\epsilon}{b M}\right)^{2}\right)^{M}\left(1+\frac{\epsilon}{b}+\frac{1}{2}\left(\frac{\epsilon}{b}\right)^{2}\right)\right)=\frac{b}{2} \ln \left(\varsigma\left(\frac{\epsilon}{b}\right)\right),
$$

where $\varsigma(x)=\left(1-\frac{x}{M}+\frac{1}{2}\left(\frac{x}{M}\right)^{2}\right)^{M}\left(1+x+\frac{x^{2}}{2}\right)$ for $x>0$. $\varsigma$ satisfies the following properties on $\mathbb{R}^{+}$:

$$
\varsigma(x)<1 \text { for } x \in] 0, M-1[\text {. }
$$


This therefore implies that

$$
\exists \epsilon \in] 0, b(M-1)\left[, \text { such that } \varsigma\left(\frac{\epsilon}{b}\right)<1,\right.
$$

and thus shows (2.22).

Proposition 2.5. If $|\mu|>\mu_{1, N}$, then the equilibrium solution with $K=1$ is asymptotically stable. If $|\mu|=\mu_{1, K}$, then the equilibrium solution with $K=1$ is unstable.

Proof. We consider $\mu$ positive but the proof for $\mu$ negative is completely similar.

Let $\mu \geq \mu_{1, N}$. We define $v^{0}$ to be the equilibrium solution and $i_{0}$ the grid point at which $v^{0}$ takes the value $v_{+}$. Recall that since $v^{0}$ is the equilibrium solution for $K=1$ and $\mu \geq \mu_{1, N}, v_{i}^{0}=v_{-}$for all $i \neq i_{0}$. Consider the minimization problem

$$
\min _{v} E(v) \text { such that } \sum_{i=0}^{N-1}\left(v_{i}-v_{i}^{0}\right)^{2} \leq \epsilon \text { and } \sum_{i=0}^{N-1} v_{i}=N \mu,
$$

with $\epsilon>0$.

We first consider all perturbations that only affect the $(N-1)$ grid points with value $v_{-}$. In this case, we can use the analysis done for $K=0$ since the mass of the $(N-1)$ perturbed grid points is preserved. We therefore know that the minimum of the energy is reached when the $(N-1)$ grid points have the same constant value $v_{-}$.

Now we consider all the perturbations that also perturb the grid point at $i_{0}$. We define $\frac{\delta_{m}}{N} \neq 0$ to be the change of mass of the $(N-1)$ remaining grid points. The value of the grid point at $i_{0}$ is now $v_{+}+\delta_{m}$. Since the value of this grid point is determined for each $\delta_{m}$, we can look at all the perturbations that change the mass of the $(N-1)$ remaining grid points by $\frac{\delta_{m}}{N}$. Here again, we can apply the analysis done for $K=0$ with the condition on the mass in equation (2.21) replaced by

$$
\frac{1}{N} \sum_{i \neq i_{0}} v_{i}=\frac{N-1}{N} v_{-}-\frac{\delta_{m}}{N},
$$

since we choose $\epsilon$ small enough so that the perturbed $(N-1)$ grid points stay strictly between $-b$ and $b$. We can therefore conclude that the energy is minimized if the $(N-1)$ remaining grid points have a constant value $v_{-}-\frac{\delta_{m}}{N-1}$. Consequently the solution that minimizes the energy with $v_{i_{0}}=v_{+}+\delta_{m}$ is

$$
v_{i}^{p}=\left\{\begin{array}{cc}
v_{+}+\delta_{m} & \text { if } i=i_{0} \\
v_{-}-\frac{\delta_{m}}{N-1} & i \neq i_{0} .
\end{array}\right.
$$

Now we consider the energy associated to the solution $v^{p}$

$$
E\left(v^{p}\right)=\frac{1}{N}\left(f\left(v_{+}+\delta_{m}\right)+(N-1) f\left(v_{-}-\frac{\delta_{m}}{N-1}\right)\right) .
$$

Differentiating with respect to $\delta_{m}$ and evaluating at $\delta_{m}=0$ gives

$$
\left.\frac{d E\left(v^{p}\right)}{d \delta_{m}}\right|_{\delta_{m}=0}=\frac{1}{N}\left(R\left(v_{+}\right)-R\left(v_{-}\right)\right)=0
$$

since $R\left(v_{-}\right)=R\left(v_{+}\right)$. Differentiating again with respect to $\delta_{m}$ and evaluating at $\delta_{m}=0$ we obtain

$$
\left.\frac{d^{2} E\left(v^{p}\right)}{d \delta_{m}^{2}}\right|_{\delta_{m}=0}=\frac{1}{N}\left(\frac{1}{N-1}-\frac{v_{-}^{2}}{b^{2}}\right) R^{\prime}\left(v_{-}\right)
$$


- Now suppose $\mu>\mu_{1, N}$.

Using Lemma 2.1 we know that $\frac{1}{N-1}-\frac{v_{-}^{2}}{b^{2}}>0$, which shows that

$$
\left.\frac{d^{2} E\left(v^{p}\right)}{d \delta_{m}^{2}}\right|_{\delta_{m}=0}>0
$$

Thus the solution $v^{0}$ is asymptotically stable.

- Now suppose $\mu=\mu_{1, N}$.

In this case, as noted at the end of Lemma 2.1, we have

$$
(N-1) v_{-}^{2}=b^{2},
$$

which leads to

$$
\left.\frac{d^{2} E\left(v^{p}\right)}{d \delta_{m}^{2}}\right|_{\delta_{m}=0}=\frac{1}{N}\left(\frac{1}{N-1}-\frac{v_{-}^{2}}{b^{2}}\right) R^{\prime}\left(v_{-}\right)=0 .
$$

Continuing to differentiate, we look at the third derivative of the energy with respect to $\delta_{m}$ and obtain

$$
\left.\frac{d^{3} E\left(v^{p}\right)}{d \delta_{m}^{3}}\right|_{\delta_{m}=0}=\frac{1}{N}\left(R^{\prime \prime}\left(v_{+}\right)-\frac{1}{(N-1)^{2}} R^{\prime \prime}\left(v_{-}\right)\right) .
$$

Using the expressions for $v_{+}$and $v_{-}$in (2.20) we obtain

$$
\left.\frac{d^{3} E\left(v^{p}\right)}{d \delta_{m}^{3}}\right|_{\delta_{m}=0}=\frac{2 \sqrt{N-1}}{b N^{3}} \frac{N-2}{N-1}=\frac{2(N-2)}{b N^{3} \sqrt{N-1}},
$$

which is strictly positive for $N \geq 3$. The steady state $v^{0}$ is therefore a saddle point in the energy landscape and thus is unstable.

REMARK 2.6. The stability analysis carried out for the one dimensional system can easily be generalized to the $d$ dimensional system, for any $d \in \mathbb{N}^{*}$, since it is only based on energy arguments which do not depend on the dimension.

\section{Upper bounds on the coarsening rate of You-Kaveh type models}

Following [8], we introduce the model density functions

$$
F_{\alpha}(x):= \begin{cases}0, & \text { if } 0 \leq|x| \leq b \\ |x-b|^{\alpha}, & \text { if }|x|>b\end{cases}
$$

where $b>0$ and $\alpha \in[0,1[$. Our results are obtained for general density functions $f \geq$ $\eta F_{\alpha}$ for some constant $\eta>0$. Without loss of generality, we work with positive initial data, i.e., $v_{i}(0) \geq 0$, for all $i \in \mathbb{L}$.

For the statement of Theorem 3.1, we introduce the following functions:

$$
\sigma=\sigma(d)= \begin{cases}2^{-\frac{4 d(1-\alpha)}{4+d(1-\alpha)}\left(\frac{d(1-\alpha)+4}{4-d(1-\alpha)}\right)^{\frac{4-d(1-\alpha)}{d(1-\alpha)+4}},}, & \text { if } 1 \leq d \leq 3, \\ 2^{-\frac{4(1-\alpha)}{2-\alpha}}\left(\frac{2-\alpha}{\alpha}\right)^{\frac{\alpha}{2-\alpha}}, & \text { if } d \geq 4\end{cases}
$$

and

$$
\rho=\rho(d)= \begin{cases}2^{\frac{d(1-\alpha)}{2}\left(\frac{d(1-\alpha)+4}{4-d(1-\alpha)}\right)^{\frac{4-d(1-\alpha)}{2 d(1-\alpha)}},}, & \text { if } 1 \leq d \leq 3, \\ 2^{2(1-\alpha)}\left(\frac{2-\alpha}{\alpha}\right)^{\frac{\alpha(1-\alpha)}{2(1-\alpha}}, & \text { if } d \geq 4 .\end{cases}
$$


THEOREM 3.1. Let $d \in \mathbb{N}^{*}$ and $f$ be the energy density function such that $f \geq \eta F_{\alpha}$ for some $\eta>0$, where $F_{\alpha}$ is defined in (3.1). Let $E$ be as in (2.6), where $v$ is the solution of (2.4). Assume $\mu>b$ and

$$
E(0)<\frac{1}{12} \frac{\eta(\mu-b)^{2-\alpha}}{\mu^{2(1-\alpha)}} .
$$

Then there exist universal constants $C_{l}<\infty$ and $C_{h}<\infty$, such that, if we let $T_{*}$ be as

$$
T_{*}=T_{*}(d)= \begin{cases}\frac{\rho}{C_{l}^{1-\alpha}} N^{d(1-\alpha)} L(0)^{\frac{4+d(1-\alpha)}{2}}, & \text { if } 1 \leq d \leq 3, \\ \frac{\rho}{C_{h}^{1-\alpha}} N^{4(1-\alpha)} L(0)^{2(2-\alpha)}, & \text { if } d \geq 4,\end{cases}
$$

we have

- If $1 \leq d \leq 3$,

$$
\frac{1}{T} \int_{0}^{T} E^{2} d t \geq \sigma C_{l}^{\frac{8(1-\alpha)}{4+d(1-\alpha)}}\left[\left(N^{4} T\right)^{-\frac{d(1-\alpha)}{d(1-\alpha)+4}}\right]^{2}
$$

provided $T \geq T_{*}$.

- If $d \geq 4$,

$$
\frac{1}{T} \int_{0}^{T} E^{2} d t \geq \sigma C_{h}^{\frac{2(1-\alpha)}{2-\alpha}}\left[\left(N^{4} T\right)^{-\frac{1-\alpha}{2-\alpha}}\right]^{2}
$$

provided $T \geq T_{*}$.

The constants $C_{l}$ and $C_{h}$ are given by the interpolation inequalities (3.4) and (3.5) respectively.

3.1. Decay relation. In this section, we establish the first ingredient for applying Kohn and Otto's technique to our problem, namely the decay relation, also called the dissipation inequality.

LEMma 3.2. $\left|\frac{d L}{d t}\right| \leq\left(-\frac{d E}{d t}\right)^{\frac{1}{2}}$.

Proof. Since the system (2.4) is gradient descent for the energy $E$ with respect to the discrete $H^{-2}$ norm, we can write

$$
v_{t}=-\nabla_{v} E,
$$

where the gradient is defined with respect to the discrete $H^{-2}$ norm. Differentiating $E$ with respect to $t$, we obtain

$$
\frac{d E}{d t}=\left\langle\nabla_{v} E, v_{t}\right\rangle_{H^{-2}}=-\left\|v_{t}\right\|_{H^{-2}}^{2} .
$$

Now differentiating $L^{2}=\|v\|_{H^{-2}}^{2}$ with respect to $t$, we obtain

$$
\left|2 L \frac{d L}{d t}\right|=2\left|\left\langle v_{t}, v\right\rangle_{H^{-2}}\right| \leq 2|| v_{t}\left\|_{H^{-2}}\right\| v\left\|_{H^{-2}}=2 L\right\| v_{t} \|_{H^{-2}},
$$

using the Cauchy-Schwartz inequality. We therefore obtain

$$
\left|\frac{d L}{d t}\right| \leq\left\|v_{t}\right\|_{H^{-2}}=\left(-\frac{d E}{d t}\right)^{\frac{1}{2}}
$$

thus proving the lemma. 
3.2. Interpolation inequality. In this section, we establish the second ingredient for applying Kohn and Otto's technique to our problem: the interpolation inequality.

Lemma 3.3. Let the length scale $L$ be defined as in (2.13) and let $\mu>b$. Assume $f \geq \eta F_{\alpha}$ for some constant $\eta>0$ and $\alpha \in[0,1[$. Let the energy $E$ be defined as in (2.6), where $v$ is the solution of (2.4), and assume

$$
E(0)<\frac{1}{12} \frac{\eta(\mu-b)^{2-\alpha}}{\mu^{2(1-\alpha)}} .
$$

Then

- If $1 \leq d \leq 3$

$$
E^{\frac{1}{1-\alpha}} L^{\frac{d}{2}} \geq \frac{C_{l}}{N^{d}}
$$

for some $C_{l}>0$ depending only on $\mu, b$ and $\alpha$.

- If $d \geq 4$

$$
E^{\frac{1}{1-\alpha}} L^{2} \geq \frac{C_{h}}{N^{4}}
$$

for some $C_{h}>0$ depending only on $\mu, b$ and $\alpha$.

We write $E_{F_{\alpha}}$, the energy associated to the energy density function $F_{\alpha}$ defined in (3.1). Note that it suffices to consider the case $f=F_{\alpha}$ since if an interpolation inequality $E_{F_{\alpha}} L^{\beta} \geq \theta>0$ holds, and $f \geq \eta F_{\alpha}$, then $E L^{\beta} \geq \eta \theta$. Similarly, the condition $E<C \eta$ implies $E_{F_{\alpha}}<C$. We therefore prove the interpolation inequality for the case $f=F_{\alpha}$. In the proof, we write $E$ for $E_{F_{\alpha}}$. As in [8], we prove the interpolation inequality in two steps and start by showing that the typical positive spike height is greater than $E^{\frac{-1}{1-\alpha}}$ in Lemma 3.4. We note that Lemma 3.4 is similar to Lemma 4 in [8], except that here we only consider the large positive spikes. If Lemma 3.4 was the direct extension of Lemma 4 in [8], we would have considered all the large spikes (i.e., both negative and positive).

Lemma 3.4. Define the typical positive spike height $h$ as

$$
h:=\left(\frac{\mu-b}{3}\right)^{\frac{1}{1-\alpha}} E^{\frac{-1}{1-\alpha}} .
$$

Define also the two sets $\mathbb{S}_{l p}$ and $\mathbb{S}_{l b}$ consisting of the large positive spikes, and points where the solution $v_{i}$ is less than $b$ (i.e., negative spikes and non spikes) respectively:

$$
\begin{aligned}
\mathbb{S}_{l p} & :=\left\{i \in \mathbb{L}: v_{i}-b>h\right\}, \\
\text { and } \quad \mathbb{S}_{l b} & :=\left\{i \in \mathbb{L}: v_{i} \leq b\right\} .
\end{aligned}
$$

Then

$$
\frac{1}{N^{d}} \sum_{i: b<v_{i} \leq b+h}\left(v_{i}-b\right) \leq \frac{\mu-b}{3}
$$

and thus

$$
\frac{1}{N^{d}} \sum_{i \in \mathbb{S}_{l p} \cup}\left(v_{i}-b\right) \geq \frac{2}{3}(\mu-b) .
$$


Proof.

$$
\begin{aligned}
\sum_{i: b<v_{i} \leq b+h}\left(v_{i}-b\right) & =\sum_{i: b<v_{i} \leq b+h}\left(v_{i}-b\right)^{\alpha}\left(v_{i}-b\right)^{1-\alpha} \\
& \leq \max _{i: b<v_{i} \leq b+h}\left(v_{i}-b\right)^{1-\alpha} \sum_{i: b<v_{i} \leq b+h}\left(v_{i}-b\right)^{\alpha} \\
& \leq h^{1-\alpha} \sum_{i: b \leq v_{i} \leq b+h} v_{i}^{\alpha} \\
& \leq N^{d} h^{1-\alpha} E=\frac{N^{d}(\mu-b)}{3} .
\end{aligned}
$$

Thus using the conservation of mass

$$
\frac{1}{N^{d}} \sum_{i \in \mathbb{S}_{l p} \cup \mathbb{S}_{l b}}\left(v_{i}-b\right)+\frac{1}{N^{d}} \sum_{i: b<v_{i} \leq b+h}\left(v_{i}-b\right)=\mu-b,
$$

we obtain

$$
\begin{aligned}
\frac{1}{N^{d}} \sum_{i \in \mathbb{S}_{l p} \cup}\left(v_{i}-b\right) & =(\mu-b)-\frac{1}{N^{d}} \sum_{i: b<v_{i} \leq b+h}\left(v_{i}-b\right) \\
& \geq \frac{2}{3}(\mu-b) .
\end{aligned}
$$

This finishes the proof.

In the proof of the interpolation inequality, we need to show that the proportion of large positive spikes is bounded from above by $\frac{\mu}{h}$. For clarity purposes, we put this result in Lemma 3.5. We note that in the second order case (see [8]), since the data $v$ is positive for all times, Lemma 3.5 is simply the Chebyshev inequality. For higher order cases however, the result is slightly more complicated to prove due to the loss of positivity in the data.

Lemma 3.5. Let the typical positive spike height $h$ be defined as in (3.6) and let the set of large positive spikes $\mathbb{S}_{l p}$ be defined as in (3.7). We define $\left|\mathbb{S}_{l p}\right|$ to be the number of large positive spikes. Then

$$
\frac{\left|\mathbb{S}_{l p}\right|}{N^{d}} \leq \frac{\mu}{h}
$$

Proof.

$$
\begin{aligned}
E & =\frac{1}{N^{d}} \sum_{i:\left|v_{i}\right| \geq b}\left|v_{i}\right|^{\alpha} \\
& \geq \frac{1}{N^{d}} \sum_{i: v_{i} \geq b+h} v_{i}^{\alpha} \\
& \geq \frac{\left|\mathbb{S}_{l p}\right|}{N^{d}}(b+h)^{\alpha} \geq \frac{\left|\mathbb{S}_{l p}\right|}{N^{d}} h^{\alpha} .
\end{aligned}
$$

Using the definition of $h$ in (3.6), we obtain

$$
h^{\frac{1}{1-\alpha}} \leq\left(\frac{\mu-b}{3}\right)^{\frac{1}{1-\alpha}}\left(\frac{N^{d}}{\left|\mathbb{S}_{l p}\right|}\right)^{\frac{1}{1-\alpha}}
$$


and thus

$$
\left|\mathbb{S}_{l p}\right| h \leq\left(\frac{\mu-b}{3}\right) N^{d} \leq \frac{\mu}{3} N^{d} \leq N^{d} \mu .
$$

Proof of Lemma 3.3. To estimate $L=\|v\|_{H^{-2}}$, we make use of its duality definition given in (2.10). In particular, recall from (2.10)

$$
L \geq \frac{\frac{1}{N^{d}} \sum_{i \in \mathbb{L}}\left(v_{i}-\mu\right) \varphi_{i}}{\|\varphi\|_{H^{2}}}
$$

where $\|\varphi\|_{H^{2}}^{2}=\frac{1}{N^{d}} \sum_{i \in \mathbb{L}}\left(\Delta_{\delta} \varphi_{i}\right)^{2}$. We also note that the smallness condition (3.3) on $E$ implies

$$
h>4^{\frac{1}{1-\alpha}} \frac{\mu^{2}}{\mu-b} .
$$

Case 1: Let $1 \leq d \leq 3$.

In this case, we use a test function $\varphi$ adapted to the large positive spikes. To construct this test function we consider a function $\mathcal{F} \in C_{c}^{\infty}\left(\mathbb{R}^{d}, \mathbb{R}^{+}\right)$, with support contained in $\left[-\frac{1}{2}, \frac{1}{2}\right]^{d}$ and $L^{1}$ norm equal to 1 , e.g.

$$
\mathcal{F}(x)= \begin{cases}C_{0} e^{\frac{-1}{\left(\frac{1}{4}-|x|^{2}\right)}} & \text { if }|x|<\frac{1}{2} \\ 0 & \text { if }|x| \geq \frac{1}{2}\end{cases}
$$

where the constant $C_{0}$ is chosen such that $\int_{\mathbb{R}^{d}} \mathcal{F}(x) d x=1$. We also let

$$
k=\left\lfloor\left(\frac{\mu-b}{4 \mu^{2}} h+1\right)\right\rfloor^{\frac{1}{d}},
$$

where $h$ is the typical positive spike height defined in (3.6). The integer $k$ is related to the radius (in number of grid points) of the cube on which the rescaled version of the function $\mathcal{F}$ will be supported.

For $p=\left(p_{1}, \cdots, p_{d}\right) \in \mathbb{S}_{l p}$ and $j \in\{1,2, \cdots, d\}$, we define the set $S_{j}^{m}$ as

$$
S_{j}^{m}:=\left\{q \in\{0,1, \cdots, N-1\}:\left|q-p_{j}\right|_{N} \leq m\right\},
$$

where $|r-s|_{N}=\min _{\ell \in \mathbb{N}}|r-s+\ell N|$ is the distance modulo $N$ between the integers $r$ and $s$ in $\{0,1, \cdots, N-1\}$. Now for $m \in \mathbb{N}^{*}$ and $p$ in $\mathbb{S}_{l p}$, we define the cube $\mathcal{Q}_{m}(p)$ as

$$
\mathcal{Q}_{m}(p):=S_{1}^{m} \times \cdots \times S_{d}^{m} .
$$

In other words, $\mathcal{Q}_{m}(p)$ is the cube of radius $m$ (in number of grid points) centered at the large positive spike located at $p \in \mathbb{S}_{l p}$. The cube $\mathcal{Q}_{m}(p)$ thus contains $(2 m+1)^{d}$ grid points. We now assume $k$ is divisible by 4 (if not, the proof follows similarly with $\frac{k}{4}$ and $\frac{k}{2}$ replaced by $\left\lfloor\frac{k}{4}\right\rfloor$ and $2\left\lfloor\frac{k}{4}\right\rfloor$ respectively), and define the two sets $\Sigma_{1}$ and $\Sigma_{2}$ as

$$
\Sigma_{1}=\bigcup_{p \in \mathbb{S}_{l_{p}}} \mathcal{Q}_{\frac{k}{4}}(p)
$$

and

$$
\Sigma_{2}=\bigcup_{p \in \mathbb{S}_{l_{p}}} \mathcal{Q}_{\frac{k}{2}}(p)
$$


$\Sigma_{1}$ is the union of all the cubes of radius $\frac{k}{4}$ centered at the large positive spikes, and $\Sigma_{2}$ is the union of all the cubes of radius $\frac{k}{2}$ centered at the large positive spikes. $\Sigma_{2}$ is therefore a dilated version of $\Sigma_{1}$, with dilation factor 2 .

We now consider the restriction of the function $\mathcal{F}$ on its support $\left[-\frac{1}{2}, \frac{1}{2}\right]^{d}$, and rescale it by a factor of $\frac{2 N}{k}$ on $\left[-\frac{1}{2}, \frac{1}{2}\right]^{d}$. The rescaled version $\mathcal{F}\left(\frac{2 N}{k} x\right)$ defined on $\left[-\frac{1}{2}, \frac{1}{2}\right]^{d}$ is then extended to $\mathbb{R}^{d}$ by periodicity with period 1 . We define $\overline{\mathcal{F}}\left(\frac{2 N}{k} x\right)$ to be the extended function. We now define the vector $\mathcal{F}$ on $\mathbb{Z}^{d}$ as

$$
\begin{aligned}
\mathcal{F}_{i} & =\left(\frac{2 N}{k}\right)^{d} N^{d} \int_{\frac{i}{N}-\frac{1}{2 N}}^{\frac{i}{N}+\frac{1}{2 N}} \overline{\mathcal{F}}\left(\frac{2 N}{k} x\right) d x, \\
& =N^{d} \int_{\frac{i}{\frac{k}{2}}-\frac{1}{2 \frac{k}{2}}}^{\frac{i}{\frac{k}{2}}+\frac{1}{2 \frac{k}{2}}} \overline{\mathcal{F}}(x) d x .
\end{aligned}
$$

The normalization factor $\left(\frac{2 N}{k}\right)^{d}$ ensures that

$$
\frac{1}{N^{d}} \sum_{i \in \mathbb{L}} \mathcal{F}_{i}=\|\mathcal{F}\|_{L^{1}\left(\mathbb{R}^{d}, \mathbb{R}^{+}\right)}=1
$$

By construction, the support of the restricted vector $\mathcal{F}$ to the lattice $\mathbb{L}$ is contained in the cube $\mathcal{Q}_{\frac{k}{4}}(0)$. Moreover since $k^{d}$ is proportional to $h$, which is an increasing quantity, we see that the size of the support of the vector $\mathcal{F}$ also increases as the system coarsens.

The test function $\varphi$ is now defined on the lattice $\mathbb{L}$, as the discrete circular convolution between the vector $\mathcal{F}$ and the characteristic function of the set $\Sigma_{1}$ :

$$
\varphi_{i}=\frac{1}{N^{d}} \sum_{j \in \mathbb{L}}\left(\chi_{\Sigma_{1}}\right)_{j} \mathcal{F}_{i-j}, \text { for } i \in \mathbb{L} .
$$

Claim:

$$
\varphi_{i}= \begin{cases}1, & \text { if } i \in \mathbb{S}_{l p} \\ 0, & \text { if } i \in\left(\stackrel{\circ}{2}_{2}\right)^{c} \\ a_{i}, & \text { else, where } 0 \leq a_{i} \leq 1 .\end{cases}
$$

For clarity purposes, the proof of the claim is provided at the end of the section. Figure 3.1 illustrates the construction of the test function $\varphi$ in the one dimensional case.

Remark 3.6. As in [8], the test function $\varphi$ is chosen to be as flat as possible, but localized enough for the term (3.14) to dominate (3.15) in the estimation of the length scale $L$.

We first bound the numerator of (3.8) from below and estimate

$$
\begin{aligned}
\frac{1}{N^{d}} \sum_{i \in \mathbb{L}} v_{i} \varphi_{i} & \geq \frac{1}{N^{d}} \sum_{i \in \mathbb{L}}\left(v_{i}-b\right) \varphi_{i} \\
& =\frac{1}{N^{d}} \sum_{i \in \mathbb{S}_{l p}}\left(v_{i}-b\right)+\frac{1}{N^{d}} \sum_{i: b<v_{i} \leq b+h}\left(v_{i}-b\right) \varphi_{i}+\frac{1}{N^{d}} \sum_{i \in \mathbb{S}_{l b}}\left(v_{i}-b\right) \varphi_{i} \\
& \geq \frac{1}{N^{d}} \sum_{i \in \mathbb{S}_{l p} \cup \mathbb{S}_{l b}}\left(v_{i}-b\right) \\
& \geq \frac{2}{3}(\mu-b) \quad \text { (using Lemma 3.4). }
\end{aligned}
$$




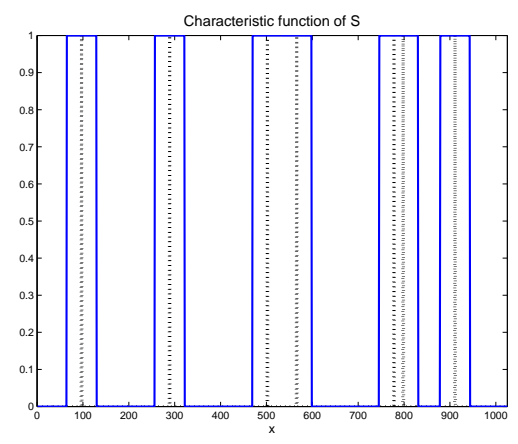

(a) Characteristic function of the set $\Sigma_{1}$. The dashed lines represent the locations of the large positive spikes.

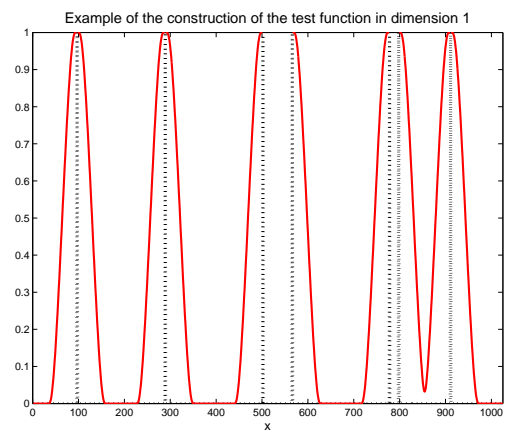

(b) Test function $\varphi$. The dashed lines represent the locations of the large positive spikes.

FIG. 3.1. One dimensional construction of the test function $\varphi$.

REMARK 3.7. The properties of $\varphi$ given in (3.13) show that the support of the test function $\varphi$ is a subset of $\Sigma_{2}$ with $\varphi$ vanishing at the boundaries of $\Sigma_{2}$. We can therefore write

$$
\varphi_{i}=\left(\chi_{\Sigma_{2}}\right)_{i}\left(\frac{1}{N^{d}} \sum_{j \in \mathcal{Q}_{\frac{k}{4}}(i)}\left(\chi_{\Sigma_{1}}\right)_{j} \mathcal{F}_{i-j}\right) \leq\left(\chi_{\Sigma_{2}}\right)_{i}, i \in \mathbb{L} .
$$

We therefore estimate

$$
\begin{aligned}
\frac{\mu}{N^{d}} \sum_{i \in \mathbb{L}} \varphi_{i} & \leq \frac{\mu}{N^{d}} \sum_{i \in \mathbb{L}}\left(\chi_{\Sigma_{2}}\right)_{i} \\
& \leq \frac{\mu}{N^{d}} \sum_{p \in \mathbb{S}_{l p}} \sum_{i \in \mathcal{Q}_{\frac{k}{2}}}(p) \\
& =\frac{\mu\left|\mathbb{S}_{l p}\right|}{N^{d}} k^{d} \\
& \leq \frac{\mu^{2}}{h} k^{d} \quad(\text { by Lemma } 3.5) \\
& \leq \frac{\mu-b}{4}+\frac{\mu^{2}}{h}\left(\text { using } k^{d} \leq \frac{\mu-b}{4 \mu^{2}} h+1 \text { from }(3.10)\right) \\
& \leq \frac{\mu-b}{4}+\left(\frac{1}{4}\right)^{\frac{1}{1-\alpha}}(\mu-b) \quad\left(\text { using } h>4^{\frac{1}{1-\alpha}} \frac{\mu^{2}}{(\mu-b)} \text { from }(3.9)\right) \\
& \leq \frac{\mu-b}{2},
\end{aligned}
$$

which combined with (3.14) gives

$$
\frac{1}{N^{d}} \sum_{i \in \mathbb{L}}\left(v_{i}-\mu\right) \varphi_{i} \geq \frac{\mu-b}{6} .
$$


We now estimate the $H^{2}$ norm of $\varphi$ and first obtain a pointwise upper bound on $\left|\Delta_{\delta} \varphi_{i}\right|$ :

$$
\begin{aligned}
& \left|\Delta_{\delta} \varphi_{i}\right|=\left|\left(\chi_{\Sigma_{2}}\right)_{i}\left(\frac{1}{N^{d}} \sum_{j \in \mathcal{Q}_{\frac{k}{4}}(i)}\left(\chi_{\Sigma_{1}}\right)_{j} \Delta_{\delta} \mathcal{F}_{i-j}\right)\right| \\
& \leq\left(\chi_{\Sigma_{2}}\right)_{i} \frac{1}{N^{d}} \sum_{j \in \mathcal{Q}_{\frac{k}{4}}(i)}\left|\left(\chi_{\Sigma_{1}}\right)_{j} \Delta_{\delta} \mathcal{F}_{i-j}\right| \\
& =\left(\chi_{\Sigma_{2}}\right)_{i}\left(\frac{2 N}{k}\right)^{2} \frac{1}{N^{d}} \sum_{j \in \mathcal{Q}_{\frac{k}{4}}(i)}\left|\left(\chi_{\Sigma_{1}}\right)_{j} \Delta_{\frac{2}{k}} \mathcal{F}_{i-j}\right| \\
& \leq\left(\chi_{\Sigma_{2}}\right)_{i}\left(\frac{2 N}{k}\right)^{2} \frac{1}{N^{d}} \sum_{j \in \mathcal{Q}_{\frac{k}{4}}(i)}\left|\Delta_{\frac{2}{k}} \mathcal{F}_{i-j}\right| \\
& =\left(\chi_{\Sigma_{2}}\right)_{i}\left(\frac{2 N}{k}\right)^{2} \underbrace{\frac{1}{N^{d}} \sum_{j \in \mathcal{Q}_{\frac{k}{4}}(0)}\left|\Delta_{\frac{2}{k}} \mathcal{F}_{j}\right|}_{\leq\|\Delta \mathcal{F}\|_{L^{1}\left(\mathbb{R}^{d}, \mathbb{R}^{+}\right)}} \\
& \leq\left(\chi_{\Sigma_{2}}\right)_{i}\left(\frac{2 N}{k}\right)^{2}\|\Delta \mathcal{F}\|_{L^{1}\left(\mathbb{R}^{d}, \mathbb{R}^{+}\right)} .
\end{aligned}
$$

For clarity, we let $C_{1}=4\|\Delta \mathcal{F}\|_{L^{1}\left(\mathbb{R}^{d}, \mathbb{R}^{+}\right)}$and estimate the $H^{2}$ norm of $\varphi$ :

$$
\begin{aligned}
\|\varphi\|_{H^{2}}^{2} & \leq \frac{1}{N^{d}} \sum_{i \in \mathbb{L}}\left(\Delta_{\delta} \varphi_{i}\right)^{2} \\
& \leq C_{1}^{2}\left(\frac{N}{k}\right)^{4} \frac{1}{N^{d}} \sum_{i \in \mathbb{L}}\left(\chi_{\Sigma_{2}}\right)_{i} \\
& \leq C_{1}^{2}\left(\frac{N}{k}\right)^{4} \frac{1}{N^{d}} \sum_{p \in \mathbb{S}_{l_{p}}} \sum_{i \in \mathcal{Q}_{\frac{k}{2}}} 1 \quad\left(\text { since } \Sigma_{2}=\bigcup_{p \in \mathbb{S}_{l_{p}}} \mathcal{Q}_{\frac{k}{2}}(p)\right) \\
& =C_{1}^{2}\left(\frac{N}{k}\right)^{4} \frac{\left|\mathbb{S}_{l p}\right|}{N^{d}} k^{d} .
\end{aligned}
$$

Thus

$$
\begin{aligned}
\|\varphi\|_{H^{2}}^{2} & \leq C_{1}^{2} N^{4} k^{d-4} \frac{\left|\mathbb{S}_{l p}\right|}{N^{d}} \\
& \leq \frac{\mu C_{1}^{2}}{h} N^{4} k^{d-4} \quad \text { (by Lemma 3.5) } \\
& \leq \frac{\mu C_{1}^{2}}{h} N^{4}\left(\frac{4 \mu^{2}}{(\mu-b) h}\right)^{\frac{4}{d}-1}\left(\text { using } k^{d} \geq \frac{\mu-b}{4 \mu^{2}} h \text { from }(3.10) \text { since } 1 \leq d<4\right) \\
& \leq \mu C_{1}^{2}\left(\frac{4 \mu^{2}}{\mu-b}\right)^{\frac{4}{d}-1} \frac{N^{4}}{h^{\frac{4}{d}}} .
\end{aligned}
$$


The final estimate is

$$
\|\varphi\|_{H^{2}} \leq \frac{\beta N^{2}}{h^{\frac{2}{d}}}
$$

with $\beta=C_{1} \sqrt{\mu\left(\frac{4 \mu^{2}}{\mu-b}\right)^{\frac{4}{d}-1}}$.

Now using the definition of $L$, we have

$$
\begin{aligned}
L=\|v\|_{H^{-2}} & \geq \frac{1}{N^{d}} \sum_{i \in \mathbb{L}}\left(v_{i}-\mu\right) \frac{\varphi_{i}}{\|\varphi\|_{H^{2}}} \\
& \geq \frac{h^{\frac{2}{d}}}{6 \beta N^{2}}(\mu-b) \quad(\text { using }(3.17) \text { and }(3.16)) \\
& \left.=\frac{1}{6 N^{2}} \frac{E^{\frac{-2}{d(1-\alpha)}}(\mu-b)^{\frac{2}{d(1-\alpha)}+1}}{3^{\frac{2}{d(1-\alpha)}} \beta} \quad \text { (using the definition of } h \text { in }(3.6)\right) \\
& =\frac{\lambda E^{\frac{-2}{d(1-\alpha)}}}{N^{2}}
\end{aligned}
$$

where $\lambda=\frac{1}{6} \frac{(\mu-b)^{\frac{2}{d(1-\alpha)}}+1}{3^{\frac{2}{d(1-\alpha)}} \beta}$. Now, letting $C_{l}=\lambda^{\frac{d}{2}}$, we can write the above inequality as

$$
E^{\frac{1}{1-\alpha}} L^{\frac{d}{2}} \geq \frac{C_{l}}{N^{d}}
$$

which proves the first part of Lemma 3.3.

REMARK 3.8. The particular profile of the test function $\varphi$ does not play much of a role in the determination of the coarsening rate. What matters is how the support of the smooth function $\mathcal{F}$ scales with $k$, and through $k, h$. In fact, any function in the space $C_{c}^{\infty}\left(\mathbb{R}^{d}, \mathbb{R}^{+}\right)$will give the same coarsening rate. We also note that this construction differs from the constructions given in [7] and [8]. In particular, it makes more transparent how the spatial dimension $d$ and the order of the equation $n$ completely determine the coarsening rate.

Case 2: Let $d \geq 4$.

In this case we define the test function $\varphi$ on the lattice $\mathbb{L}$ as the characteristic function of the set of large positive spikes $\mathbb{S}_{l p}$, namely $\varphi=\chi_{\mathbb{S}_{l_{p}}}$. We remark that contrary to the low dimensional case $(1 \leq d \leq 3)$, the test function is chosen to be supported on single spikes. We first estimate its $H^{2}$ norm

$$
\begin{aligned}
\|\varphi\|_{H^{2}}^{2} & \leq \frac{8\left|\mathbb{S}_{l p}\right| d}{N^{d-4}} \\
& \left.\leq \frac{8 d N^{4} \mu}{h} \quad \text { (using Lemma } 3.5\right)
\end{aligned}
$$

and obtain

$$
\|\varphi\|_{H^{2}} \leq 2 N^{2} \sqrt{\frac{2 d \mu}{h}}
$$


Now we estimate

$$
\begin{aligned}
\frac{1}{N^{d}} \sum_{i \in \mathbb{L}}\left(v_{i}-\mu\right) \varphi_{i} & =\frac{1}{N^{d}} \sum_{i \in \mathbb{S}_{l_{p}}}\left(v_{i}-\mu\right) \\
& \geq \frac{1}{N^{d}} \sum_{i \in \mathbb{S}_{l_{p}}}\left(v_{i}-b\right)-\mu \frac{\left|\mathbb{S}_{l p}\right|}{N^{d}} \\
& \geq \frac{1}{N^{d}} \sum_{i \in \mathbb{S}_{l p} \cup \mathbb{S}_{l b}}\left(v_{i}-b\right)-\frac{\mu^{2}}{h}(\text { using Lemma 3.5) } \\
& \geq \frac{2}{3}(\mu-b)-\frac{\mu^{2}}{h} \quad(\text { using Lemma 3.4) } \\
& \geq \frac{2}{3}(\mu-b)-\frac{\mu-b}{4} \quad\left(\text { using } h>4^{\frac{1}{1-\alpha}} \frac{\mu^{2}}{(\mu-b)} \text { from }(3.9)\right) \\
& \geq \frac{5}{12}(\mu-b) .
\end{aligned}
$$

Therefore

$$
\frac{1}{N^{d}} \sum_{i \in \mathbb{L}}\left(v_{i}-\mu\right) \varphi_{i} \geq \frac{5}{12}(\mu-b) .
$$

Combining estimates (3.19) and (3.20), we obtain

$$
\begin{aligned}
L=\|v\|_{H^{-2}} & \geq \frac{1}{\|\varphi\|_{H^{2}} N^{d}} \sum_{i \in \mathbb{L}}\left(v_{i}-\mu\right) \varphi_{i} \\
& \geq \frac{5(\mu-b)}{24 \sqrt{2 d} N^{2}} \sqrt{\frac{h}{\mu}} \\
& \left.=5 \frac{(\mu-b)^{\frac{3-2 \alpha}{2(1-\alpha)}} E^{\frac{-1}{2(1-\alpha)}}}{24 \sqrt{6 d \mu} N^{2}} \text { (using the definition of } h \text { in }(3.6)\right) .
\end{aligned}
$$

Letting $C_{h}=\frac{25(\mu-b)^{\frac{3-2 \alpha}{1-\alpha}}}{3456 d \mu}$, we can write the above inequality as

$$
E^{\frac{1}{1-\alpha}} L^{2} \geq \frac{C_{h}}{N^{4}},
$$

which proves the second part of Lemma 3.3.

Combining the decay relation with the interpolation inequality, and using Kohn and Otto's ODE argument [16], we obtain the result of Theorem 3.1.

Proof of the claim. By construction, $0 \leq \varphi_{i} \leq 1$ for all $i \in \mathbb{L}$. We have $\varphi_{i} \geq 0$ because it is the sum of positive quantities, and $\varphi_{i} \leq 1$ because the vector $\mathcal{F}$ is normalized to sum up to 1 . Also we note that $\varphi_{i}$ can be written as

$$
\varphi_{i}=\frac{1}{N^{d}} \sum_{j \in \mathcal{Q}_{\frac{k}{4}}(i)}\left(\chi_{\Sigma_{1}}\right)_{j} \mathcal{F}_{i-j},
$$

since the support of the restricted vector $\mathcal{F}$ to the lattice $\mathbb{L}$ is contained in the cube $\mathcal{Q}_{\frac{k}{4}}(0)$. 
- Now suppose $i \in \mathbb{S}_{l p}$. Since $\Sigma_{1}=\bigcup_{p \in \mathbb{S}_{l p}} \mathcal{Q}_{\frac{k}{4}}(p)$, we know that $\mathcal{Q}_{\frac{k}{4}}(i) \subset \Sigma_{1}$, which implies $\left(\chi_{\Sigma_{1}}\right)_{j}=1$ for $j \in \mathcal{Q}_{\frac{k}{4}}(i)$. Thus $\varphi_{i}$ simplifies to

$$
\varphi_{i}=\frac{1}{N^{d}} \sum_{j \in \mathcal{Q}_{\frac{k}{4}}(i)} \mathcal{F}_{i-j}=\frac{1}{N^{d}} \sum_{j \in \mathcal{Q}_{\frac{k}{4}}(0)} \mathcal{F}_{j}=\frac{1}{N^{d}} \sum_{j \in\left\{-\frac{N}{2},-\frac{N}{2}+1, \cdots, \frac{N}{2}\right\}^{d}} \mathcal{F}_{j}=1 .
$$

- Now suppose $i \in\left(\stackrel{\circ}{\Sigma}_{2}\right)^{c}$. Since $\Sigma_{2}$ is a dilated version by 2 of $\Sigma_{1}$, we have by construction

$$
\text { For all } i \in\left(\stackrel{\circ}{\Sigma}_{2}\right)^{c} \text { and } j \in \Sigma_{1},\left|i_{q}-j_{q}\right|_{N} \geq \frac{k}{4}, \text { for all } 1 \leq q \leq d .
$$

Therefore, if $i \in\left({\stackrel{\circ}{\Sigma_{2}}}^{c}\right.$, we have $\mathcal{Q}_{\frac{k}{4}}(i) \subset\left(\Sigma_{1}\right)^{c}$ which implies $\left(\chi_{\Sigma_{1}}\right)_{j}=0$ for $j \in \mathcal{Q}_{\frac{k}{4}}(i)$. Thus

$$
\varphi_{i}=\frac{1}{N^{d}} \sum_{j \in Q_{\frac{k}{4}}(i)}\left(\chi_{\Sigma_{1}}\right)_{j} \mathcal{F}_{i-j}=0 .
$$

3.3. Rigorous result in terms of the spike density. In this section, we establish the rigorous result of Theorem 3.1 in terms of the spike density. In other words, we show that for a certain interval of time, namely until the spike density gets low, the time-average of the spike density is bounded from below by a function of time that decays with the rate indicated by the one obtained in Theorem 3.1.

We introduce the following notation. Let $K(t)$ be the number of spikes at time $t$, and let $T_{*}$ be as in (3.2). Define also $I(T)$ to be

$$
I(T)=\frac{1}{T} \int_{0}^{T}\left(\frac{K(t)}{N^{d}}\right)^{2(1-\alpha)} d t .
$$

Corollary 3.9. Let $\gamma \in] 0,1[$. Assume there exists a constant $\theta>0$ such that $f(x) \leq$ $\theta|x|^{\alpha}$ for all $|x|$ large enough, and assume also that $\mu$ is large enough such that $f(\mu) \leq$ $\theta \mu^{\alpha}$. If $I\left(T_{*}\right)>\gamma$, then define $T^{*}$ to be the first time at which $I$ equals $\gamma$, i.e.,

$$
T^{*}=\min \left\{t \geq T_{*}: I(t)=\gamma\right\} .
$$

Then for $T \in\left[T_{*}, T^{*}\right]$ and under the assumptions of Theorem 3.1, there exist constants $C_{1}<\infty$ and $C_{2}<\infty$, such that

- If $1 \leq d \leq 3$,

- If $d \geq 4$,

$$
I(T) \geq C_{1}\left[\left(N^{4} T\right)^{-\frac{d}{d(1-\alpha)+4}}\right]^{2(1-\alpha)} .
$$

$$
I(T) \geq C_{2}\left[\left(N^{4} T\right)^{-\frac{1}{2-\alpha}}\right]^{2(1-\alpha)} .
$$

Proof. Let $\mathbb{S}$ be the set of spikes and suppose $I\left(T_{*}\right)>\gamma$, which by definition implies $I(T) \geq \gamma$ for $T \in\left[T_{*}, T^{*}\right]$. We define the energy of the spikes $E_{S}$ to be

$$
E_{S}:=\frac{1}{N^{d}} \sum_{i \in \mathbb{S}} f\left(v_{i}\right)
$$


and the energy of the non-spikes $E_{N S}$ to be

$$
E_{N S}:=\frac{1}{N^{d}} \sum_{i \in \mathbb{L} \backslash \mathbb{S}} f\left(v_{i}\right)
$$

where $f$ is the energy density function. Then $E=E_{N S}+E_{S}$. We also define the mass of the spikes to be $\mu_{S}:=\frac{1}{N^{d}} \sum_{i \in \mathbb{S}} v_{i}$ and the mass of the non-spikes to be $\mu_{N S}:=$ $\frac{1}{N^{d}} \sum_{i \in \mathbb{L} \backslash \mathbb{S}} v_{i}$. We easily see that

$$
E_{N S}=\frac{1}{N^{d}} \sum_{i:\left|v_{i}\right|<b} f\left(v_{i}\right) \leq f(b)
$$

For $E_{S}$, we have

$$
E_{S} \leq \max \left\{E_{S}: \frac{1}{N^{d}} \sum_{i \in \mathbb{S}} v_{i}=\mu_{S}\right\}
$$

Using the concavity of the function $f$ for $|x|>b$, we can show that the maximum of the energy $E_{S}$ is reached when all the spikes have the same height $\left|v_{i}\right|=\frac{N^{d} \mu_{S}}{K}$, assuming their mass $\mu_{S}$ is fixed. Moreover, since the heights of the non-spikes is always greater than $-b$, we can bound their mass $\mu_{N S}$ from below as

$$
\mu_{N S} \geq-\left(1-\frac{K}{N^{d}}\right) b
$$

and deduce that

$$
\frac{N^{d} \mu_{S}}{K} \leq \frac{N^{d}}{K}(\mu+b)-b .
$$

Therefore

$$
\begin{aligned}
E & \leq f(b)+\frac{K}{N^{d}} f\left(\frac{N^{d}}{K}(\mu+b)-b\right) \\
& \leq f(b)+\theta \frac{K}{N^{d}}\left(\frac{N^{d}}{K}(\mu+b)-b\right)^{\alpha}\left(\text { since } \frac{N^{d}}{K}(\mu+b)-b>\mu\right) \\
& \leq f(b)+\theta \frac{K}{N^{d}}\left(2 \frac{N^{d}}{K} \mu\right)^{\alpha}(\text { since } b+\mu<2 \mu) \\
& =f(b)+(2 \mu)^{\alpha} \theta\left(\frac{K}{N^{d}}\right)^{1-\alpha} .
\end{aligned}
$$

Squaring both sides, we obtain

$$
E^{2} \leq 2\left(f(b)^{2}+(2 \mu)^{2 \alpha} \theta^{2}\left(\frac{K}{N^{d}}\right)^{2(1-\alpha)}\right)
$$

whose time-average satisfies

$$
I(T)=\frac{1}{T} \int_{0}^{T}\left(\frac{K}{N^{d}}\right)^{2(1-\alpha)} d t \geq \frac{1}{\lambda_{1}(\theta) \mu^{2 \alpha}}\left(\frac{1}{T} \int_{0}^{T} E^{2} d t-2 f(b)^{2}\right),
$$


where $\lambda_{1}(\theta)=2^{2 \alpha+1} \theta^{2}$. If $T \in\left[T_{*}, T^{*}\right]$, we have by assumption that $I(T) \geq \gamma$, which implies

$$
\frac{2 f(b)^{2}}{\lambda_{1}(\theta) \mu^{2 \alpha}} \leq \lambda_{2}(\gamma) I(T)
$$

with $\lambda_{2}(\gamma)=\frac{2 f(b)^{2}}{\gamma \lambda_{1}(\theta) \mu^{2 \alpha}}$. Moreover, if $T \in\left[T_{*}, T^{*}\right]$, we can apply Theorem 3.1 and therefore obtain

$$
I(T) \geq \begin{cases}\frac{\sigma C_{l}^{\frac{8(1-\alpha)}{4+d(1-\alpha)}}}{\left(1+\lambda_{2}(\gamma)\right) \lambda_{1}(\theta) \mu^{2 \alpha}}\left[\left(N^{4} T\right)^{-\frac{d(1-\alpha)}{d(1-\alpha)+4}}\right]^{2}, & \text { if } 1 \leq d \leq 3, \\ \frac{\sigma C_{h}^{\frac{2(1-\alpha)}{2-\alpha}}}{\left(1+\lambda_{2}(\gamma)\right) \lambda_{1}(\theta) \mu^{2 \alpha}}\left[\left(N^{4} T\right)^{-\frac{1-\alpha}{2-\alpha}}\right]^{2}, & \text { if } d \geq 4 .\end{cases}
$$

This finishes the proof.

REMARK 3.10. Since $I\left(T^{*}\right)=\gamma$, we can bound $T^{*}$ from below by

$$
T^{*} \geq \frac{1}{N^{4}} \begin{cases}T_{l}^{0}=\left(\frac{\sigma C_{l}^{\frac{8(1-\alpha)}{4+d(1-\alpha)}}}{2\left(4^{\alpha} \mu^{2 \alpha} \theta^{2} \gamma+f(b)^{2}\right)}\right)^{\frac{d(1-\alpha)+4}{2 d(1-\alpha)}}, & \text { if } 1 \leq d \leq 3, \\ T_{h}^{0}=\left(\frac{\sigma C_{h}^{\frac{2(1-\alpha)}{2-\alpha}}}{2\left(4^{\alpha} \mu^{2 \alpha} \theta^{2} \gamma+f(b)^{2}\right)}\right)^{\frac{2-\alpha}{2(1-\alpha)}}, & \text { if } d \geq 4 .\end{cases}
$$

For $\mu$ large, $C_{l}(3.18)$ scales like $\mu^{\frac{2 \alpha+d(1-\alpha)}{2(1-\alpha)}}$ and $C_{h}$ (3.21) scales like $\mu^{\frac{2-\alpha}{1-\alpha}}$, which implies

$$
T_{l}^{0} \sim\left(\frac{\mu^{\frac{4(2 \alpha+d(1-\alpha))}{4+d(1-\alpha)}}}{\gamma \mu^{2 \alpha}+f(b)^{2}}\right)^{\frac{d(1-\alpha)+4}{2 d(1-\alpha)}} \text { and } T_{r}^{0} \sim\left(\frac{\mu^{2}}{\gamma \mu^{2 \alpha}+f(b)^{2}}\right)^{\frac{2-\alpha}{2(1-\alpha)}} .
$$

Since $\mu$ is fixed, we can choose $\gamma>0$ such that $\gamma \mu^{2 \alpha}=O(1)$ and obtain

$$
T_{l}^{0} \sim \mu^{\frac{2(2 \alpha+d(1-\alpha))}{d(1-\alpha)}} \text { and } T_{r}^{0} \sim \mu^{\frac{2-\alpha}{1-\alpha}} .
$$

Since both exponents $\frac{2(2 \alpha+d(1-\alpha))}{d(1-\alpha)}$ and $\frac{2-\alpha}{1-\alpha}$ are greater or equal to 2 for any $\alpha \in\left[0,1\left[\right.\right.$, we see that $T_{l}^{0}$ and $T_{r}^{0}$ can be very large. Thus $T^{*}$ can be very large, which implies that Corollary 3.9 can be valid for a very long time.

\section{Upper bounds on coarsening for the $2 n$-th order equations}

4.1. Equations and scheme. More generally, we study the following $2 n$-th order equation

$$
v_{t}=(-1)^{n+1} \Delta^{n}(R(v)),
$$

for $n \in \mathbb{N}^{*}$. We note that for $n$ even, equation (4.1) is closely related to equation (1.4)

$$
u_{t}=-\Delta^{\frac{n}{2}}\left(R\left(\Delta^{\frac{n}{2}} u(x)\right)\right) .
$$

Taking the Laplacian raised to $\frac{n}{2}$ of both sides of equation (1.4) and setting $v=\Delta^{\frac{n}{2}} u$, we see that $v$ satisfies (4.1) if $u$ satisfies (1.4). For $n$ odd, the connection is only true 
in the one dimensional case. To see that let's recall the one dimensional equation $(1.4)$

$$
u_{t}=\partial_{x}^{n} R\left(\partial_{x}^{n} u(x)\right)
$$

Taking $n$ partial derivatives in $x$ of both sides of equation (4.2) and setting $v=\partial_{x}^{n} u$, we see that $v$ satisfies the one dimensional equation (4.1) if $u$ satisfies (4.2).

We now discretize $(4.1)$ on the same grid $[0,1]^{d}$, and consider the system $v_{i}$ satisfying the system of ODEs

$$
\frac{d v_{i}}{d t}=(-1)^{n+1}\left(\Delta_{\delta}\right)^{n}\left(R\left(v_{i}\right)\right), i \in \mathbb{L}
$$

We recall that we use periodic boundary conditions. Like (2.4), the system (4.3) is gradient descent for the energy $E$ defined in (2.6) with respect to the discrete $H^{-n}$ norm

$$
\|v\|_{H^{-n}}:=\left\{\begin{array}{c}
\left\{\sup _{\phi} \frac{1}{N^{d}} \sum_{i \in \mathbb{L}}\left(v_{i}-\mu\right) \phi_{i}: \frac{1}{N^{d}} \sum_{i \in \mathbb{L}}\left(\left(\Delta_{\delta}\right)^{\frac{n}{2}} \phi_{i}\right)^{2} \leq 1\right\} \\
\left\{\sup _{\phi} \frac{1}{N^{d}} \sum_{i \in \mathbb{L}}\left(v_{i}-\mu\right) \phi_{i}: \frac{1}{N^{d}} \sum_{i \in \mathbb{L}}\left|\left(\nabla_{\delta} \Delta_{\delta}^{\frac{n-1}{2}}\right) \phi_{i}\right|^{2} \leq 1\right\} \text { is even, } n \text { is odd. }
\end{array}\right.
$$

Like (2.4), if the initial data has some mass in the ill-posed regime, the evolution of (4.3) gives rise to spikes whose coarsening behavior is very similar to the coarsening of (2.4). Thus we define our length scale quantity $L$ as

$$
L:=\|v\|_{H^{-n}}
$$

When chosen this way, we see that $L$ scales with the number of spikes $K$ as

$$
L \sim \begin{cases}\frac{1}{K_{1}^{\frac{n}{d}}} & \text { for } 1 \leq d \leq 2 n-1, \\ \frac{1}{\sqrt{K}} & \text { for } d \geq 2 n .\end{cases}
$$

4.2. Main result. We introduce the following functions

$$
\tilde{\sigma}=\tilde{\sigma}(d)= \begin{cases}2^{-\frac{4 d(1-\alpha)}{2 n+d(1-\alpha)}\left(\frac{d(1-\alpha)+2 n}{2 n-d(1-\alpha)}\right)^{\frac{2 n-d(1-\alpha)}{d(1-\alpha)+2 n}},} & \text { if } 1 \leq d \leq 2 n-1, \\ 2^{-\frac{4(1-\alpha)}{2-\alpha}}\left(\frac{2-\alpha}{\alpha}\right)^{\frac{\alpha}{2-\alpha}}, & \text { if } d \geq 2 n,\end{cases}
$$

and

$$
\tilde{\rho}=\tilde{\rho}(d)= \begin{cases}2^{\frac{d(1-\alpha)}{n}\left(\frac{d(1-\alpha)+2 n}{2 n-d(1-\alpha)}\right)^{\frac{2 n-d(1-\alpha)}{2 d(1-\alpha)}},} & \text { if } 1 \leq d \leq 2 n-1, \\ 2^{2(1-\alpha)}\left(\frac{2-\alpha}{\alpha}\right)^{\frac{\alpha}{2(1-\alpha)}}, & \text { if } d \geq 2 n\end{cases}
$$

THEOREM 4.1. Let $d \in \mathbb{N}^{*}$ and let $f$ be the energy density function such that $f \geq \eta F_{\alpha}$ for some $\eta>0$, where $F_{\alpha}$ is defined in (3.1). Let $E$ be as in (2.6) where $v$ is the solution of (4.3). Assume $\mu>b$ and

$$
E(0)<\frac{1}{12} \frac{\eta(\mu-b)^{2-\alpha}}{\mu^{2(1-\alpha)}} .
$$


Then there exist universal constants $\tilde{C}_{l}<\infty$ and $\tilde{C}_{h}<\infty$, such that, if we let $\tilde{T}_{*}$ be as

$$
\tilde{T}_{*}=\tilde{T}_{*}(d)= \begin{cases}\frac{\tilde{\rho}}{{\tilde{C_{l}}}_{l}^{1-\alpha}} N^{d(1-\alpha)} L(0)^{\frac{2 n+d(1-\alpha)}{n}}, & \text { if } 1 \leq d \leq 2 n-1, \\ \widetilde{\tilde{C}_{h}^{1-\alpha}} N^{2 n(1-\alpha)} L(0)^{2(2-\alpha)}, & \text { if } d \geq 2 n,\end{cases}
$$

we have

- If $1 \leq d \leq 2 n-1$,

$$
\frac{1}{T} \int_{0}^{T} E^{2} d t \geq \tilde{\sigma} \tilde{C}_{l}^{\frac{4 n(1-\alpha)}{2 n+d(1-\alpha)}}\left[\left(N^{2 n} T\right)^{-\frac{d(1-\alpha)}{d(1-\alpha)+2 n}}\right]^{2},
$$

provided $T \geq \tilde{T}_{*}$.

- If $d \geq 2 n$,

$$
\frac{1}{T} \int_{0}^{T} E^{2} d t \geq \tilde{\sigma} \tilde{C}_{h}^{\frac{2(1-\alpha)}{2-\alpha}}\left[\left(N^{2 n} T\right)^{-\frac{1-\alpha}{2-\alpha}}\right]^{2}
$$

provided $T \geq \tilde{T}_{*}$.

The constants $\tilde{C}_{l}$ and $\tilde{C}_{h}$ are given by the interpolation inequalities (4.6) and (4.7) respectively.

4.3. Decay relation and interpolation inequality. To prove Theorem 4.1, we use the same argument as in the fourth order case, and establish a decay relation and an interpolation inequality. The decay relation remains the same as in the fourth order case since it only relies on the gradient descent property of the scheme. The difference lies in the interpolation inequality.

Lemma 4.2. Let the length scale $L$ be defined as in (4.4) and let $\mu>b$. Assume $f \geq \eta F_{\alpha}$ for some constant $\eta>0$ and $\alpha \in[0,1[$. Let the energy $E$ be defined as in (2.6) where $v$ is the solution of (4.3), and assume

$$
E(0)<\frac{1}{12} \frac{\eta(\mu-b)^{2-\alpha}}{\mu^{2(1-\alpha)}} .
$$

Then

- If $1 \leq d \leq 2 n-1$

$$
E^{\frac{1}{1-\alpha}} L^{\frac{d}{n}} \geq \frac{\tilde{C}_{l}}{N^{d}}
$$

for some $\tilde{C}_{l}>0$ depending only on $\mu, b, n$ and $\alpha$.

- If $d \geq 2 n$

$$
E^{\frac{1}{1-\alpha}} L^{2} \geq \frac{\tilde{C_{h}}}{N^{2 n}}
$$

for some $\tilde{C}_{h}>0$ depending only on $\mu, b, n$ and $\alpha$.

Proof. This proof very closely follows the proof of Lemma 3.3. In particular, we keep all the definitions introduced there, like the positive spike height $h$ and the number of grid points $k$. Lemmas 3.4 and 3.5 therefore remain true. We also note that the only difference between the general case and the fourth order case is in the definition of $L$. In particular, the choice of test functions remains the same as in the fourth order case. Thus, estimates (3.16) and (3.20) still hold. 
- Let $1 \leq d \leq 2 n-1$.

We define the test function $\varphi$ as in (3.12) since the function $\mathcal{F}$ is infinitely differentiable, and estimate the $H^{n}$ norm of $\varphi$ as in the fourth order case. In particular, $\left|\Delta_{\delta} \varphi_{i}\right|$ is replaced by $\left|\left(\Delta_{\delta}^{\frac{n}{2}}\right) \varphi_{i}\right|$ if $n$ is even or $\left|\left(\nabla_{\delta} \Delta_{\delta}^{\frac{n-1}{2}}\right) \varphi_{i}\right|$ if $n$ is odd. The final estimate is

$$
\|\varphi\|_{H^{n}}^{2} \leq \frac{\tilde{\beta} N^{n}}{h^{\frac{2 n}{d}}},
$$

where $h$ is defined in (3.6) and $\tilde{\beta}$ is a constant depending only on $\mu, b, n$ and $\mathcal{F}$. Combining (4.8) with (3.16), we obtain

$$
L=\|v\|_{H^{-n}} \geq \frac{\tilde{\lambda} E^{-\frac{n}{d(1-\alpha)}}}{N^{2 n}},
$$

where $\tilde{\lambda}=\frac{1}{6} \frac{(\mu-b)^{\frac{2}{d(1-\alpha)}}+1}{3^{\frac{2}{d(1-\alpha)} \tilde{\beta}}}$. Letting $\tilde{C}_{l}=\tilde{\lambda}^{\frac{d}{n}}$, we obtain

$$
E^{\frac{1}{1-\alpha}} L^{\frac{d}{n}} \geq \frac{\tilde{C}_{l}}{N^{d}}
$$

- Let $d \geq 2 n$.

We consider the discrete function $\varphi=\chi_{\mathbb{S}_{l_{p}}}$ as in the fourth order case. An estimate on its $H^{n}$ norm gives

$$
\|\varphi\|_{H^{n}}^{2} \leq \frac{\tau(n)\left|\mathbb{S}_{l p}\right| d}{N^{d-2 n}}
$$

where $\tau(n)$ is a constant depending only on the order of derivatives. It can be worked out exactly but its exact value does not affect the bound on the coarsening rate. Using the result of Lemma 3.5 we obtain

$$
\|\varphi\|_{H^{n}}^{2} \leq \frac{\tau(n) d N^{2 n} \mu}{h}
$$

Combining estimate (3.20) with (4.9) we show that

$$
L=\|v\|_{H^{-n}} \geq \frac{5(\mu-b)^{\frac{3-2 \alpha}{2(1-\alpha)}} E^{-\frac{1}{2(1-\alpha)}}}{12 N^{n} \sqrt{3 \tau d \mu}} .
$$

Letting $\tilde{C_{h}}=\frac{25(\mu-b)^{\frac{3-2 \alpha}{1-\alpha}}}{432 \tau d \mu}$, we can write the above inequality as

$$
E^{\frac{1}{1-\alpha}} L^{2} \geq \frac{\tilde{C_{h}}}{N^{2 n}}
$$

This finishes the proof of Lemma 4.2.

We now establish the analogue of Corollary 3.9 and let $\tilde{T}_{*}$ be as in (4.5).

Corollary 4.3. Let $I(T)$ be as in (3.22) and define $\tilde{T}^{*}$ as in Corollary 3.9, with $T_{*}$ replaced by $\tilde{T}_{*}$. Then under the assumptions of Theorem 4.1 and Corollary 3.9, for $T \in\left[\tilde{T}_{*}, \tilde{T}^{*}\right]$, there exist constants $\tilde{C}_{1}<\infty$ and $\tilde{C}_{2}<\infty$ such that 
- If $1 \leq d \leq 2 n-1$,

$$
I(T) \geq \tilde{C}_{1}\left[\left(N^{2 n} T\right)^{-\frac{d}{d(1-\alpha)+2 n}}\right]^{2(1-\alpha)} .
$$

- If $d \geq 2 n$,

$$
I(T) \geq \tilde{C}_{2}\left[\left(N^{2 n} T\right)^{-\frac{1}{2-\alpha}}\right]^{2(1-\alpha)} .
$$

The proof is analogous to the one of Corollary 3.9.

\section{Numerical evidence and discussion}

In this section, we present numerical experiments for the fourth order (i.e., YouKaveh) and sixth order equation corroborating the rigorous statement of Corollary 4.3, and discuss some of the implications of this result in image processing applications.

5.1. Numerical results. We show the actual coarsening rate of (4.1) by discretizing the system of ODEs (4.3) in time and solving it numerically as in $[7,8]$. The graphs below show that the coarsening rate depends on $N$ as our bounds indicate. We also note that our analysis does not include the early time dynamics since our results only hold for later times. We let $G\left(v_{i}^{n}\right)=-\Delta_{h}^{n}\left(R\left(v_{i}^{n}\right)\right)$ and use the forward Euler method in time

$$
\frac{v_{i}^{n+1}-v_{i}^{n}}{\delta t}=G\left(v_{i}^{n}\right)
$$

As in [8], we choose the following density function $f$

$$
f(x)=\left(1+x^{2}\right)^{\frac{\alpha}{2}},
$$

with $\alpha=\frac{1}{2}$. As an initial condition, we use a perturbation of $v_{i}=2$. Using the result of Corollary 3.9 and Corollary 4.3, we have

- In the fourth order case

$$
\frac{1}{T} \int_{0}^{T}\left(\frac{K}{N^{d}}\right)^{2-2 \alpha} d t \geq C\left\{\begin{array}{lr}
\left(\left(N^{4} T\right)^{\frac{-d}{d(1-\alpha)+4}}\right)^{2-2 \alpha}, & \text { for } 1 \leq d \leq 3 \\
\left(\left(N^{4} T\right)^{\frac{-1}{2-\alpha}}\right)^{2-2 \alpha}, & \text { for } d \geq 4
\end{array}\right.
$$

for some constant $C<\infty$.

- In the sixth order case

$$
\frac{1}{T} \int_{0}^{T}\left(\frac{K}{N^{d}}\right)^{2-2 \alpha} d t \geq C\left\{\begin{array}{lr}
\left(\left(N^{6} T\right)^{\frac{-d}{d(1-\alpha)+6}}\right)^{2-2 \alpha}, & \text { for } 1 \leq d \leq 5 \\
\left(\left(N^{6} T\right)^{\frac{-1}{2-\alpha}}\right)^{2-2 \alpha}, & \text { for } d \geq 6
\end{array}\right.
$$

for some constant $C<\infty$.

In the figures below we show loglog plots of the spike density $\frac{K}{N^{d}}$ of the solution of (5.1) versus $N^{2 n} T$, with $f$ defined in (5.2), for $n=2$ and $n=3$, in support of the rigorous result stated in Corollary 4.3. The rigorous bounds from Theorem 4.1 and Corollary 4.3 also lead to the following heuristic scalings for the length scale $L$

$$
N^{n} L \sim \begin{cases}\left(N^{2 n} T\right)^{\frac{n}{d(1-\alpha)+2 n}}, & \text { if } 1 \leq d \leq 2 n-1, \\ \left(N^{2 n} T\right)^{\frac{1}{2(2-\alpha)}}, & \text { if } d \geq 2 n .\end{cases}
$$




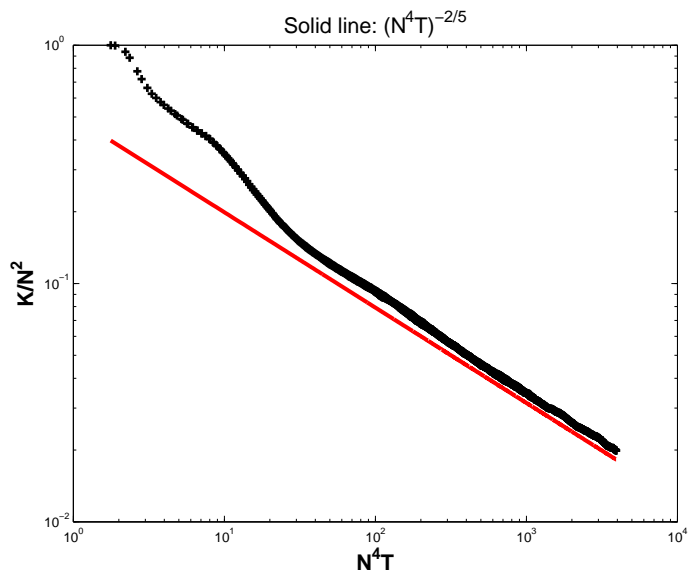

FIG. 5.1. Experiments done with the two-dimensional You-Kaveh equation (2.4) with $f(x)=$ $\left(1+x^{2}\right)^{\frac{1}{4}}$. The spike density $\frac{K}{N^{2}}$ is represented by the dashed lines for $N=175$ and $N=200$. The coarsening rate $\left(N^{4} T\right)^{-\frac{2}{5}}$ indicated by the bound obtained in Theorem 3.1 is represented by the solid line. After an initial period, the spikes appear to coarsen at the predicted rate indicating that the bound seems close to optimal.

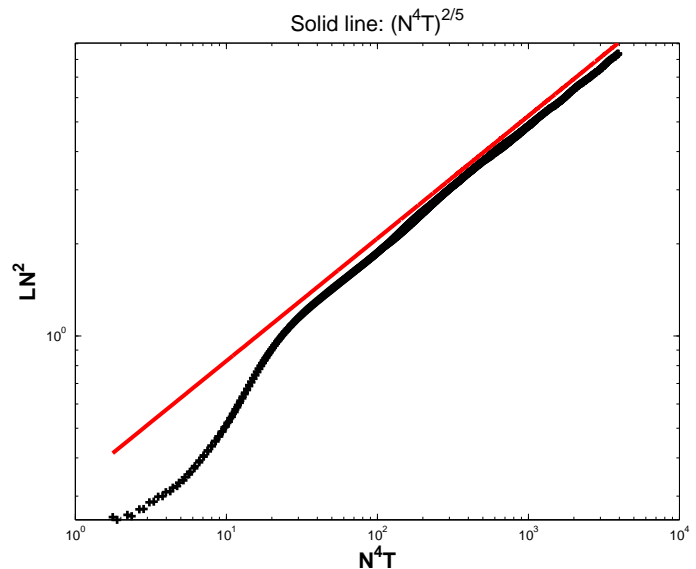

FIG. 5.2. Experiments done with the two-dimensional You-Kaveh equation (2.4) with $f(x)=$ $\left(1+x^{2}\right)^{\frac{1}{4}}$. The rate $\left(N^{4} T\right)^{\frac{2}{5}}$ is represented by the solid line. The quantity $L N^{2}$, represented by the dashed lines for $N=175$ and $N=200$, appears to coarsen at the rate $\left(N^{4} T\right)^{\frac{2}{5}}$ after an initial period of time. This shows that after a transient initial time, the length scale $L$ behaves like $L \sim \frac{1}{K}$, validating our choice (2.13) as a length scale measure.

We provide plots for the length scale $L$ to corroborate the above scalings. For the fourth order equation, our numerical results are presented in dimensions two, three, four and five to illustrate the fact that the coarsening rate does indeed become constant after dimension four. Figures 5.1, 5.3, 5.5, and 5.7 show the decay of the spike density $\frac{K}{N^{d}}$ versus $N^{2 n} T$, superimposed with the theoretical coarsening rate 


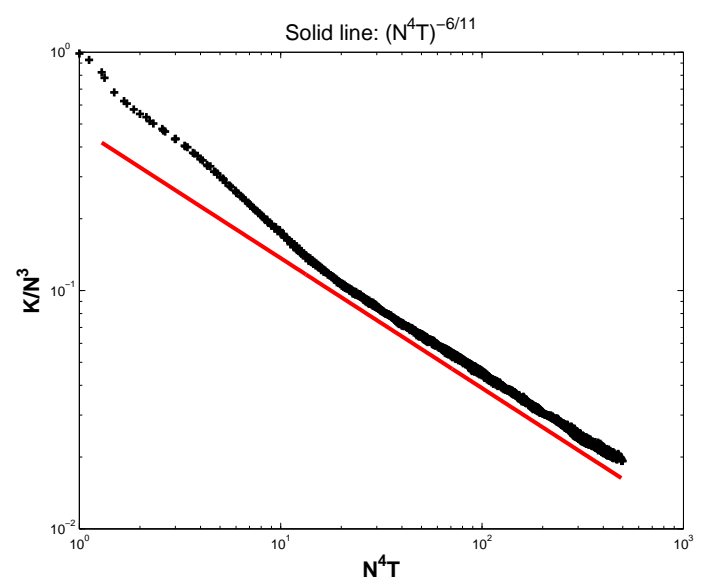

FIG. 5.3. Experiments done with the three-dimensional You-Kaveh equation (2.4) with $f(x)=$ $\left(1+x^{2}\right)^{\frac{1}{4}}$. The spike density $\frac{K}{N^{3}}$ is represented by the dashed lines for $N=20$ and $N=25$. The coarsening rate $\left(N^{4} T\right)^{-\frac{6}{11}}$ indicated by the bound obtained in Theorem 3.1 is represented by the solid line. After an initial period, the spikes appear to coarsen at the predicted rate indicating that the bound seems close to optimal.

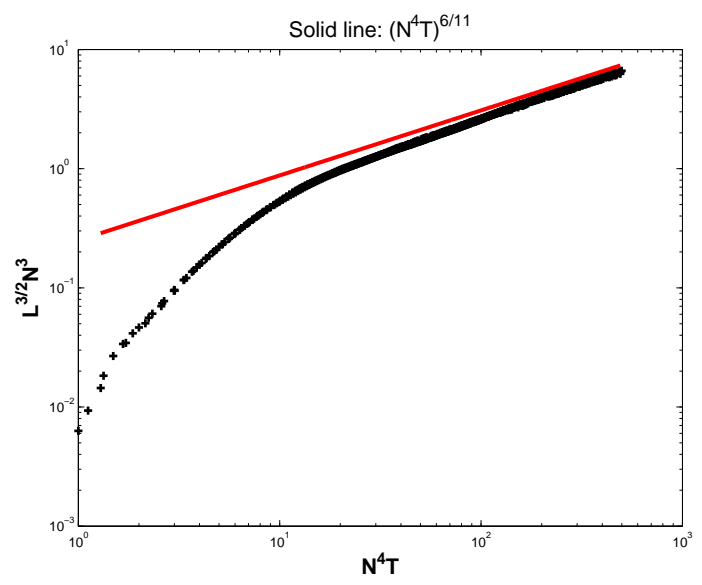

FIG. 5.4. Experiments done with the three-dimensional You-Kaveh equation (2.4) with $f(x)=$ $\left(1+x^{2}\right)^{\frac{1}{4}}$. The rate $\left(N^{4} T\right)^{\frac{6}{11}}$ is represented by the solid line. The quantity $L^{\frac{3}{2}} N^{3}$, represented by the dashed lines for $N=20$ and $N=25$, appears to coarsen at the rate $\left(N^{4} T\right)^{\frac{6}{11}}$ after an initial period of time. This shows that after a transient initial time, the length scale $L$ behaves like $L \sim \frac{1}{K^{\frac{2}{3}}}$, validating our choice (2.13) as a length scale measure.

$\left(N^{2 n} T\right)^{-\frac{d}{d(1-\alpha)+2 n}}$ in dimensions two, three, four and five respectively. Figures 5.2, $5.4,5.6$, and 5.8 display similar plots for the length scale $L$. The last two figures illustrate our numerical results for the sixth order equation in dimension two. Figure 5.9 shows the plot for the spike density $\frac{K}{N^{d}}$ and figure 5.10 the plot for the length scale $L$. 


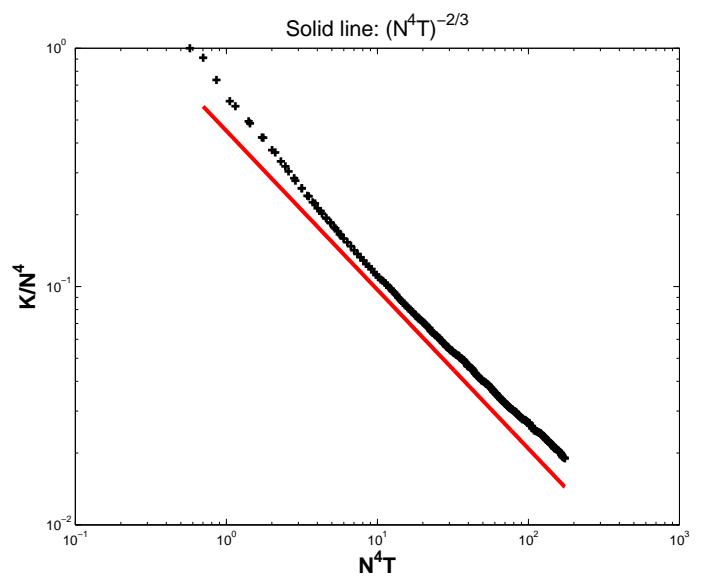

FIG. 5.5. Experiments done with the four-dimensional You-Kaveh equation (2.4) with $f(x)=$ $\left(1+x^{2}\right)^{\frac{1}{4}}$. The spike density $\frac{K}{N^{4}}$ is represented by the dashed lines for $N=10$ and $N=15$. The coarsening rate $\left(N^{4} T\right)^{-\frac{2}{3}}$ indicated by the bound obtained in Theorem 3.1 is represented by the solid line. After an initial period, the spikes appear to coarsen at the predicted rate indicating that the bound seems close to optimal.

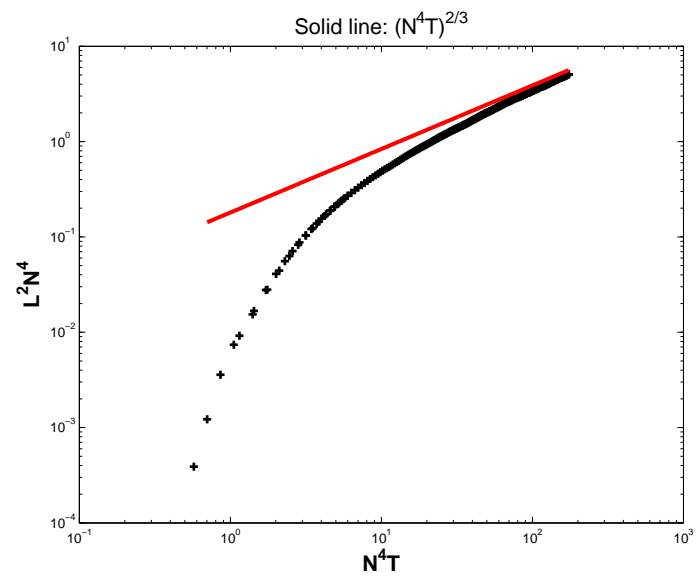

FIG. 5.6. Experiments done with the four-dimensional You-Kaveh equation (2.4) with $f(x)=$ $\left(1+x^{2}\right)^{\frac{1}{4}}$. The rate $\left(N^{4} T\right)^{\frac{2}{3}}$ is represented by the solid line. The quantity $L^{2} N^{4}$, represented by the dashed lines for $N=10$ and $N=15$, appears to coarsen at the rate $\left(N^{4} T\right)^{\frac{2}{3}}$ after an initial period of time. This shows that after a transient initial time, the length scale $L$ behaves like $L \sim \frac{1}{\sqrt{K}}$, validating the choice (2.13) as a length scale measure.

The computational results presented below show very good agreement with the theoretical bounds of sections 3 and 4 and their implications described above. In particular, although the rigorous results presented in this paper are one-sided bounds, as in previous applications of the Kohn and Otto's technique, they seem to reflect the typically observed behavior of the dynamics. Indeed, after a brief initial period of rapid change, in our experiments both the spike density $\frac{K}{N^{d}}$ and the length scale 


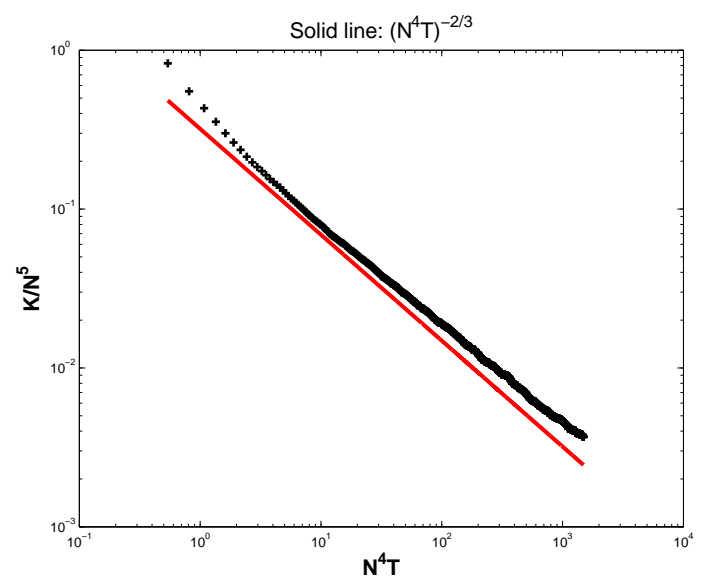

FIG. 5.7. Experiments done with the five-dimensional You-Kaveh equation (2.4) with $f(x)=$ $\left(1+x^{2}\right)^{\frac{1}{4}}$. The spike density $\frac{K}{N^{5}}$ is represented by the dashed lines for $N=7$. The coarsening rate $\left(N^{4} T\right)^{-\frac{2}{3}}$ indicated by the bound obtained in Theorem 3.1 is represented by the solid line. After an initial period, the spikes appear to coarsen at the predicted rate indicating that the bound seems close to optimal and corroborating the fact that the coarsening rate becomes constant after dimension four.

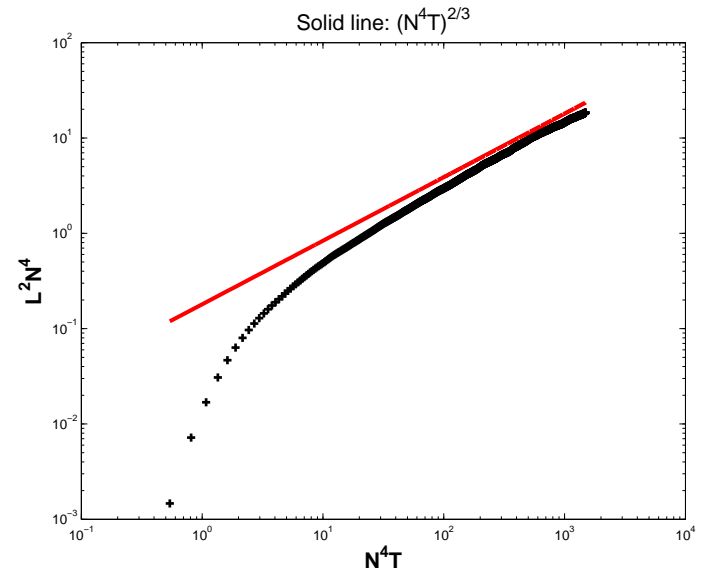

FIG. 5.8. Experiments done with the five-dimensional You-Kaveh equation (2.4) with $f(x)=$ $\left(1+x^{2}\right)^{\frac{1}{4}}$. The rate $\left(N^{4} T\right)^{\frac{2}{3}}$ is represented by the solid line. The quantity $L^{2} N^{4}$, represented by the dashed lines for $N=7$, appears to coarsen at the rate $\left(N^{4} T\right)^{\frac{2}{3}}$ after an initial period of time. This shows that after a transient initial time, the length scale $L$ behaves like $L \sim \frac{1}{\sqrt{K}}$, as in the four dimensional case.

quantity $L$ settle into a rate that is remarkably close to the one-sided bounds. Nevertheless, a slight deviation from the bounds is present in the behavior of $\frac{K}{N^{d}}$ at dimensions $d \geq 4$ for the fourth order (i.e. You-Kaveh) equation (see figures 5.5 and 5.7 ), whereas no such deviation can be discerned in our experiments in the behavior of $L$ (see figures 5.6 and 5.8). A similar slight deviation was observed in [7] for the 


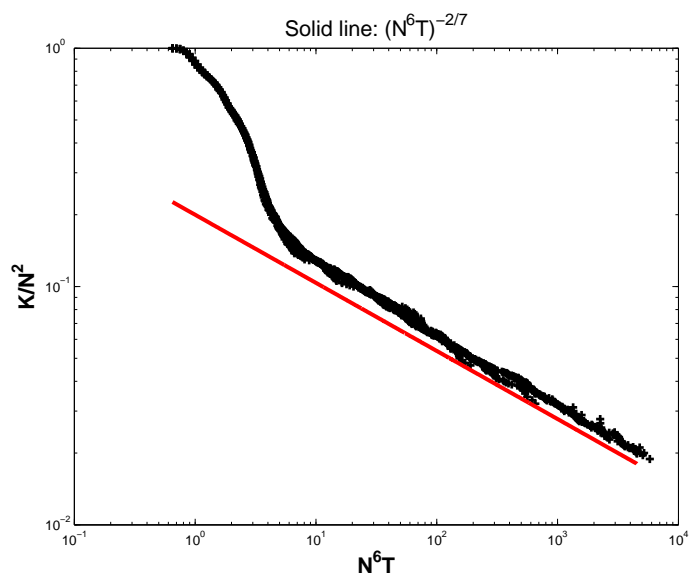

FIG. 5.9. Experiments done with the two-dimensional sixth-order equation (4.3) with $f(x)=$ $\left(1+x^{2}\right)^{\frac{1}{4}}$. The spike density $\frac{K}{N^{2}}$ is represented by the dashed lines for $N=30, N=50$ and $N=75$. The coarsening rate $\left(N^{6} T\right)^{-\frac{2}{7}}$ indicated by the bound obtained in Theorem 4.1 is represented by the solid line. After an initial period, the spikes appear to coarsen at the predicted rate indicating that the bound seems close to optimal.

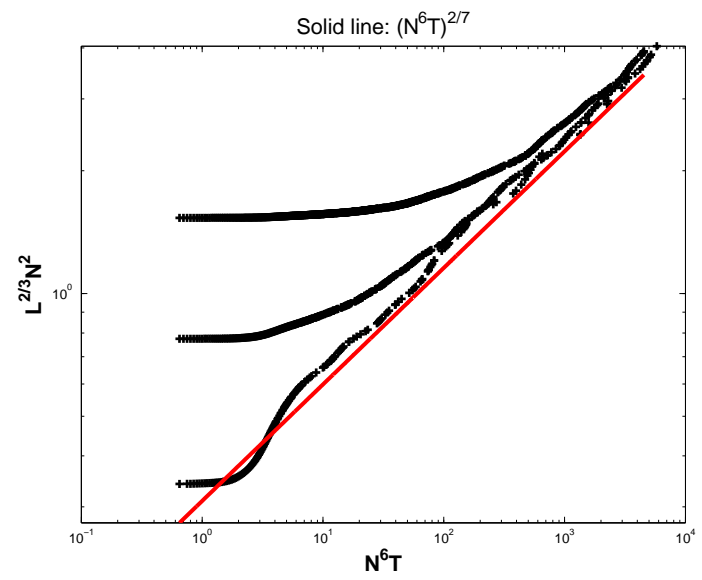

FIG. 5.10. Experiments done with the two-dimensional sixth order equation (4.3) with $f(x)=$ $\left(1+x^{2}\right)^{\frac{1}{4}}$. The rate $\left(N^{6} T\right)^{\frac{2}{7}}$ is represented by the solid line. The quantity $L^{\frac{2}{3}} N^{2}$, represented by the dashed lines for $N=30, N=50$ and $N=75$, appears to coarsen at the rate $\left(N^{6} T\right)^{\frac{2}{7}}$ after an initial period of time. This shows that after a transient initial time, the length scale $L$ behaves like $L \sim \frac{1}{K^{\frac{3}{2}}}$, validating the choice (4.4) as a length scale measure.

second order (i.e. Perona-Malik) equation at dimension $d=2$. Based on these cases, it may be reasonable to suspect that in general, for the $2 n$-th order equation, the deviation appears at dimension $d=2 n$, which is when the interpolation inequality of Lemma 4.2 switches from one form to the other. 
5.2. Discussion. In practical applications to image processing of the schemes considered in this paper (e.g., the You-Kaveh model (2.4)), a central question is the appropriate choice of parameters. In particular, given an image to be simplified (i.e., coarsened), it is often critical to know when to stop the time evolution of the processing equation. Since the complexity of an image is generally measured in terms of its "edge content", Corollary 4.3 presented in section 4 may be interpreted to furnish a partial answer to this question. Although the results of this paper cannot provide a universal, absolute value for the time at which a desired level of simplification in the image will be reached, they can be used to infer a scaling between the evolution time and the level of simplification: indeed, inverting the rigorous statement for the spike density given in Corollary 4.3, we arrive at the following

$$
\begin{cases}T \sim\left(\frac{K}{N^{d}}\right)^{-\frac{d(1-\alpha)+2 n}{d}}, & \text { if } 1 \leq d \leq 2 n-1, \\ T \sim\left(\frac{K}{N^{d}}\right)^{-(2-\alpha)}, & \text { if } d \geq 2 n,\end{cases}
$$

where $T$ is the time of evolution and $\frac{K}{N^{d}}$ the spike density. An interesting facet of formula (5.3) is its dependence on $n$, the order of the equation up to a factor of two. We see that in low dimensions, namely $1 \leq d \leq 2 n-1$ (two, three and four being the most common in image processing), the coarsening rate (in terms of the exponent) slows down as the order of the equation increases. In particular, we see that in dimensions one, two and three, the fourth order You-Kaveh evolution leads to a slower simplification of the image than the second order Perona-Malik equation, potentially requiring a longer integration time. This observation concerns the continuous in time versions of the schemes, and is thus independent of the choice of time-stepping method used for the fully discrete system. It is an additional factor that needs to be taken into account when assessing the computational complexity of the various models, together with the usual stability restriction on the time step size that occurs in explicit schemes (i.e., the CFL condition which gets worse as the order of the equation increases). At this junction, it is worth mentioning the observation in [7] that implicit schemes for these ill-posed evolutions do not seem to yield the expected improvements in complexity: although implicit schemes can indeed be unconditionally stable, thus allowing for larger time steps, decreasing the number of spikes by a certain factor requires roughly the same number of time steps as an explicit scheme, regardless of the step size. A reasonable explanation for this observation is that large time steps cannot capture accurately enough the evolutions of spikes (a highly non smooth solution) and thus introduce drastic errors that alter the discrete simulations. There is therefore no real gain in processing speed by using implicit time-stepping methods for these equations. Nevertheless, despite their additional, inherent computational complexity, these You-Kaveh type models find use in image processing due to the improved quality of their results. There is indeed a trade-off between computational time and quality of the processed image, but the final choice of which element is more important (i.e., computational speed versus quality of the results) is in the end up to the practitioner and dependent on the application.

\section{Conclusion}

We obtained rigorous upper bounds on the coarsening rate of a family of discretein-space, ill-posed, nonlinear diffusion equations of any even degree. This paper focused most specifically on the fourth order case which corresponds to a family of equations proposed by You and Kaveh. Their goal was to address some of the issues of the well-known Perona-Malik model, such as staircasing. Our bounds were 
obtained for a wide class of diffusivities and differ from the bounds obtained in $[7,8]$. Besides, contrary to $[7,8]$ where the rigorous bounds were written for the energy only, we established rigorous bounds for both the energy and the spike density.

This paper focuses on the fourth order You-Kaveh diffusion equations since they have applications in computer vision and image processing where they are used as edge preserving image denoising models. The evolution of these equations on a noisy picture forces the image to gradually simplify by merging neighboring regions while preserving their edges sharp. Understanding the coarsening rate of these equations is therefore an important step towards the automatic determination of the stopping time of their evolution for a given level of image simplification.

Numerical experiments indicate that the upper bounds we established are attained, and generally observed.

Acknowledgement. The author would like to thank her advisor Selim Esedoḡlu for proposing the project and providing help and guidance throughout the preparation of this paper.

\section{REFERENCES}

[1] H. Amann, Time-delayed Perona-Malik type problems, Acta. Math. Univ. Comeniance, LXXVI, 15-38, 2007.

[2] A. Belahmidi and A. Chambolle, Time-delay regularization of anisotropic diffusion, ESAIM Mathematical Modelling and Numerical Analysis, 39(2), 231-251, 2005.

[3] G. Bellettini and G. Fusco, A regularized Perona-Malik functional: some aspects of the gradient dynamics, H. Broer, J. Mahwin, A. Vanderbauwhede and S.V. Lunel (eds), EQUADIFF 2003 Proceedings of the International Conference on Differential Equations, Hasselt, Belgium, July, 2003.

[4] G. Bellettini, M. Novaga and E. Paolini, Global solutions to the gradient flow equation of a nonconvex functional, SIAM J. Math. Anal., 37(5), 1657-1687, 2006.

[5] F. Catt, P.L. Lions, J.M. Morel and T. Coll, Image selective smoothing and edge detection by nonlinear diffusion, SIAM J. Numer. Anal., 29, 182-193, 1992.

[6] S. Esedoḡu, An analysis of the Perona-Malik scheme, Commun. Pure Appl. Math, 54, 14421487, 2001.

[7] S. Esedoḡlu and J. Greer, Upper bounds of the coarsening rate of discrete ill-posed nonlinear diffusion equations, Commun. Pure Appl. Math., 62(1), 57-81, 2009.

[8] S. Esedoḡlu and D. Slepčev, Refined upper bounds of the coarsening rate of discrete ill-posed nonlinear diffusion equations, Nonlinearity, 21(1), 2759-2776, 2008.

[9] M. Ghisi and M. Gobbino, A class of local classical solutions for the one-dimensional PeronaMalik equation, preprint, 2006.

[10] M. Ghisi and M. Gobbino, Gradient estimates for the Perona-Malik equation, Math. Ann., 337(3), 557-590, 2007.

[11] M. Gobbino, Entire solutions of the one-dimensional Perona-Malik equation, Commun. Part. Differ. Equ., 32(4-6), 719-743, 2007.

[12] J. Goodman, A. Kurganov and P. Rosenau, Breakdown in Burgers-type equations with saturating dissipation fluxes, Nonlinearity, 12, 247-268, 1999.

[13] J. Greer and A. Bertozzi, Traveling wave solutions of fourth order PDEs for image processing, SIAM J. Math. Anal., 36(1), 38-68, 2004.

[14] B. Kawohl and N. Kutev, Maximum and comparison principle for one-dimensional anisotropic diffusion, Math. Ann., 311, 107-123, 1998.

[15] S. Kichenassamy, The Perona-Malik paradox, SIAM J. Appl. Math., 57(5), 1328-1342, 1997.

[16] R.V. Kohn and F. Otto, Upper bounds on coarsening rates, Commun. Math. Phys., 229(3), 375-395, 2002.

[17] R.V. Kohn and X. Yan, Upper bound on the coarsening rate for an epitaxial growth model, Commun. Pure Appl. Math., 56(11), 1549-1564, 2003.

[18] M. Nitzberg and T. Shiota, Nonlinear image filtering with edge and corner enhancement, IEEE Trans. Pattern Anal. Machine Intell., 14, 629-639, 1992.

[19] F. Otto, T. Rump and D. Slepčev, Coarsening rates for a droplet model: rigorous upper bounds, SIAM J. Math. Anal., 32(12), 503-529, 2006. 
[20] P. Perona and J. Malik, Scale space and edge detection using anisotropic diffusion, Technical report, Dept. of EECS, U.C. Berkeley, 1987.

[21] P. Perona and J. Malik, Scale-space and edge detection using anisotropic diffusion, IEEE Trans. Pattern. Anal. Machine Intell., 12, 629-639, 1990.

[22] P. Perona, J. Malik and T. Shiota, Geometry-Driven diffusion in computer vision, chapter Anisotropic diffusion, Kluwer, 73-92, 1995.

[23] T.P. Witelski, D.G. Schaeffer and M. Shearer, A discrete model for an ill-posed nonlinear parabolic PDE, Phys. D., 160(3-4), 189-221, 2001.

[24] Y.L. You and M. Kaveh, Fourth-order partial differential equations for noise removal, IEEE Transactions on Image Processing, 9(10), 1723-1730, 2000. 